\title{
əConfiguring LES Based on Dropsonde Data in Sparsely Sampled Areas in the Subtropical Atlantic
}

\author{
Stephanie Reilly, Sara Dal Gesso, a And Roel Neggers \\ University of Cologne, Institute for Geophysics and Meteorology, Cologne, Germany
}

(Manuscript received 16 January 2019, in final form 3 September 2019)

\begin{abstract}
This study explores the question of how field campaign data, gathered over a large area that is poorly sampled, can be used to make large-eddy simulation (LES) realizations more representative of the local conditions. To this purpose, dropsonde data recorded during the first Next-Generation Aircraft Remote Sensing for Validation Studies (NARVAL)-South campaign, in the marine subtropical North Atlantic, are blended into the forcing data. Control simulations are driven by time-dependent forcings derived from a combination of analyses and short-range weather forecasts, using weak nudging to prevent excessive model drift. A second set of simulations is driven by forcing data with dropsonde profiles included at the time points of their release. Metrics are designed to (i) quantify the impact on the boundary layer vertical profiles as a result of nudging toward the dropsondes and (ii) use a probabilistic method to allow a fair comparison of the inversion strengths in the simulations and observations. The simulations show strong time variation in the cloud-layer depth on relatively short time scales, which is commensurate with recent observational studies in the area. Nudging toward dropsondes improves the representation of the atmospheric profiles throughout the depth of the boundary layer in all simulations. However, the impact on the inversion strength is less pronounced. All impacts persist for some time after the dropsonde time point, depending on the intensity of the nudging and the nudging time window. The sensitivity of the results to nudging details and vertical resolution is assessed.
\end{abstract}

\section{Introduction}

Subtropical marine trade wind boundary layer (BL) clouds play a critical role in Earth's climate system given their strong impact on the vertical transport of heat, humidity, and momentum, and their significant impact on the radiative energy budget (Bony et al. 2015). Uncertainties in predictions of future climate have been associated with poor representations of BL clouds in models through cloud-climate feedbacks that are still not fully understood (Bony and Dufresne 2005; Tan et al. 2016; Blossey et al. 2016). A long-standing and continuing scientific and practical problem in atmospheric science is that $\mathrm{BL}$ clouds need to be parameterized in general circulation models (GCMs) (e.g., IPCC 2013).

¿ Denotes content that is immediately available upon publication as open access.

${ }^{\text {a }}$ Current affiliation: AMIGO s.r.l, Rome, Italy.

Corresponding author: Stephanie Reilly, sreilly@uni-koeln.de
High-resolution models, such as large-eddy simulation (LES) models, can be used to simulate BL clouds (Lilly 1966; Deardorff 1972; Sommeria 1976). The four-dimensional fields provided by LES give an insight into BL clouds and help improve their parameterization for GCMs. In an attempt to determine the strengths and weaknesses in LES models, many intercomparison studies have been carried out focusing on a variety of atmospheric conditions. Initially these intercomparison studies focused on stratocumulus clouds (e.g., Moeng et al. 1996; Duynkerke et al. 1999; Ackerman et al. 2009). More recent intercomparison studies have also included studying the representation of shallow cumulus cases (e.g., Brown et al. 2002; Siebesma et al. 2003; vanZanten et al. 2011). Studies over the past few years have focused on BL cloud responses to changes in climate (e.g., Bellon and Stevens 2012; Rieck et al. 2012; Tan et al. 2016; Blossey et al. 2016).

While LES models have been shown to provide realistic realizations of BL cloud fields, the technique does have some well-known shortcomings. Those reported often include numerical and/or physical problems. Some examples include a lack of performance near 
strong inversions (e.g., Stevens et al. 1999; Gentine et al. 2015) or having a fully parameterized cloud microphysics scheme. A more practical difficulty is related to the configuration of LES experiments based on field campaign data (Stevens et al. 2001). Often such data are scarce over the oceans.

Recent field campaigns in the subtropical Atlantic, featuring state-of-the-art instrumentation, can provide observations that have the potential to fill this data gap. One example, the Rain in Cumulus over the Ocean (RICO) campaign (Rauber et al. 2007) took place in 2004 and 2005 near Barbados. While RICO coincides geographically with the permanently operational Barbados Cloud Observatory (BCO) site, a more recent field campaign has taken place that was designed to start filling the data gap over the remote quarters of the subtropical Atlantic Ocean. The Next-Generation Aircraft Remote Sensing for Validation Studies (NARVAL)South campaign (Klepp et al. 2014) took place in December 2013 and featured the High Altitude and Long Range Research Aircraft (HALO) as its main instrument platform.

The NARVAL data complement the RICO/BCO dataset, by providing a more complete coverage of cumulus in the trades. Most LES experiments of trade wind cumulus have focused on the Barbados area (Siebesma et al. 2003; vanZanten et al. 2011; Seifert and Heus 2013), with fewer experiments carried out for the upstream areas. There have been a few exceptions, for example Stevens et al. (2001). Unfortunately, the NARVAL dataset is much less extensive compared to the rich RICO/BCO datasets, with HALO only briefly present in areas of interest. This potentially complicates the ability to build an LES case. How to overcome this problem is an open research question and is applicable to all field campaigns similar to NARVAL. It is therefore scientifically attractive to build LES cases based on the dropsonde (DS) observations recorded during NARVAL.

This study aims to investigate the potential for using DS data, recorded during field campaigns, to make LES realizations more representative of the atmospheric conditions observed in the region. There is a focus on the BL deep structure in particular. In situ DS data, as obtained during the NARVAL campaign, are ideal for this study due to its relatively homogeneous largescale conditions, in humidity and potential temperature, over a large area in the northeastern Atlantic trade wind subsidence regime. A second benefit of using the NARVAL dataset are the additional cloud remote sensing datasets recorded with the HALO Microwave Package (HAMP), which complement the DS data.
Our main objective is to investigate to what extent it is possible to use limited DS data to build an LES realization that is realistic to a reasonable degree and subsequently useful for gaining insight into observed trade wind cumulus cloud fields. To this purpose a total of 36 LES experiments were performed to coincide with dropsondes. These include a control series purely driven by the Integrated Forecasting System (IFS) data from the European Centre for Medium-Range Weather Forecasts (ECMWF), a second series in which DS data are merged, and an additional series of sensitivity tests to investigate the impact of numerics and the nudging details.

The observations, LES model, and large-scale forcings used in this study are introduced in section 2 . Section 3 describes the general strategy, the setup of the model and the nudging, along with the details on the two main sets of simulations. The results are presented in section 4 , which documents the general behavior of the simulations while also investigating and quantifying the impact of including DSs in the nudging. The focus of the results lies on the BL deep structure and the inversion strength. In addition, the sensitivity to details in the nudging are assessed. Finally, sections 5 and 6 summarizes the successes and shortcomings in the study followed by the main conclusions and outlook.

\section{Observations and models}

\section{a. NARVAL campaign}

The first NARVAL campaign took place throughout December 2013 and January 2014, with HALO functioning as the main instrument platform. The campaign was divided into two parts: NARVAL-South, based off the coast of Barbados and focused on the North Atlantic trade wind region, and NARVAL-North, based in Iceland and focused on the North Atlantic storm track (Klepp et al. 2014). This study focuses on NARVALSouth, which took place between 10 and 22 December 2013 and consisted of eight research flights. Four of these flights were transatlantic flights between Oberpfaffenhofen in Germany and Barbados. The four additional flights were local flights out of Barbados (Klepp et al. 2014; Schnitt et al. 2017).

The target area of NARVAL-South was the subtropical Atlantic trade wind region, which in general is not routinely sampled by state-of-the-art meteorological instrumentation. The exception to this rule is the $\mathrm{BCO}$ on the island of Barbados (Stevens et al. 2016) and the Eastern North Atlantic (ENA) site (Lamer et al. 2015). Therefore, this campaign recorded important 
TABLE 1. Location and time of the dropsonde (DS) launches.

\begin{tabular}{|c|c|c|c|c|c|c|c|c|c|c|c|}
\hline & DS01 & $\mathrm{DS} 02$ & DS03 & DS04 & DS05 & DS06 & DS07 & DS08 & DS09 & DS10 & DS11 \\
\hline Launch Time (UTC) & 1408 & 1504 & 1546 & 1600 & 1622 & 1706 & 1724 & 1827 & 1909 & 1947 & 2001 \\
\hline Lat $\left({ }^{\circ} \mathrm{N}\right)$ & 14.3 & 16.6 & 18.0 & 18.1 & 15.4 & 18.4 & 18.8 & 16.9 & 15.4 & 14.0 & 13.5 \\
\hline Lon $\left({ }^{\circ} \mathrm{E}\right)$ & 304.2 & 311.7 & 317.0 & 318.7 & 319.4 & 319.0 & 320.8 & 312.8 & 307.7 & 303.3 & 301.6 \\
\hline
\end{tabular}

information on the atmospheric conditions in the North Atlantic trade wind region.

Two observational datasets are used in this study. Vaisala DSs, which gathered in situ measurements, and retrievals from HAMP. Over the course of the eight research flights, a total of 75 DSs were launched (Klepp et al. 2014; Schnitt et al. 2017). The DSs used during NARVAL-South contain a global positioning system (GPS), which was initially developed in the 1990 s as a result of a collaboration between the National Center for Atmospheric Research (NCAR), the National Oceanic and Atmospheric Administration (NOAA), and the German Aerospace Research Establishment (DLR) (Hock and Franklin 1999). These DSs record profiles for a variety of different atmospheric variables, including pressure with an accuracy of $0.5 \mathrm{hPa}$, relative humidity $(\mathrm{RH})$ with an accuracy of $2 \%$, specific humidity $q_{v}$, potential temperature $\Theta$, and virtual potential temperature $\Theta_{v}$. The wind speed in the west-east direction $u$ and north-south direction $v$ were recorded from the GPS system on the DS and have an accuracy of $0.5-2.0 \mathrm{~m} \mathrm{~s}^{-1}$. The HAMP instrument is a microwave radiometer consisting of 26 channels between 22 and 183.31 GHz (Mech et al. 2014; Konow et al. 2018; Jacob et al. 2019). The retrievals of integrated water vapor (IWV) used in this study are obtained using the seven K-band channels along with the $90-\mathrm{GHz}$ channel (Jacob et al. 2019).

This study focuses on Research Flight 4 (RF04), which took place on 14 December 2013. RF04 left Barbados and flew approximately $2000 \mathrm{~km}$ across the North Atlantic before returning to Barbados. During this flight 11 DSs were launched, the details of which are given in Table 1. The flight path can be seen in Fig. 1, with the locations of each of the DS launched shown by the circles. RF04 was chosen due to the relatively steady state and uniform large-scale conditions (Stevens et al. 2016) and the presence of a well-defined convective BL including persistent fair-weather cumulus.

In order understand the atmospheric conditions observed by the DSs the recorded profiles of $\Theta_{v}, q_{v}$, and $\mathrm{RH}$ are shown in Fig. 2. Within the BL there is some variation in the profiles for $\Theta_{v}$. However, all DSs record $\Theta_{v}$ within a 5-K range at the surface. The BL for each of the $\Theta_{v}$ profiles is capped by an inversion that occurs between 1.5 and $2.5 \mathrm{~km}$ for nearly all DSs. Farther aloft, the variation among the $\Theta_{v}$ profiles is much less pronounced. The lapse rate above the BL indicates relatively stable atmospheric conditions. The profiles of $q_{v}$ and RH, shown in Figs. 2b and 2c, respectively, are more variable in the BL compared to the $\Theta_{v}$ profiles. This is emphasized in the region of the capping inversion at the top of the BL.

For the individual DS profiles, the capping inversions are more pronounced in the humidity profiles compared to those in the $\Theta_{v}$ profiles. While the BL capping inversion in the $\Theta_{v}$ profiles occur at relatively similar altitudes for each DS, this is not the case in the humidity profiles. The capping inversions in the humidity profiles predominantly occur between 0.7 and $1.7 \mathrm{~km}$. One outlying profile, DS11, has a capping inversion at approximately $4 \mathrm{~km}$. This DS recorded a region of deep convection, which was also noted by Stevens et al. (2016).

One common trait between each of the DS humidity profiles is the extremely dry conditions above the BL, also observed by Stevens et al. (2016). The dry conditions can be seen in Figs. $2 b$ and 2c, where there appears to be little to no humidity present above the inversion.

\section{b. LES model}

The Dutch Atmospheric Large-Eddy Simulation (DALES) model, formerly known as the KNMI LES model, was used to run LESs over the locations of the

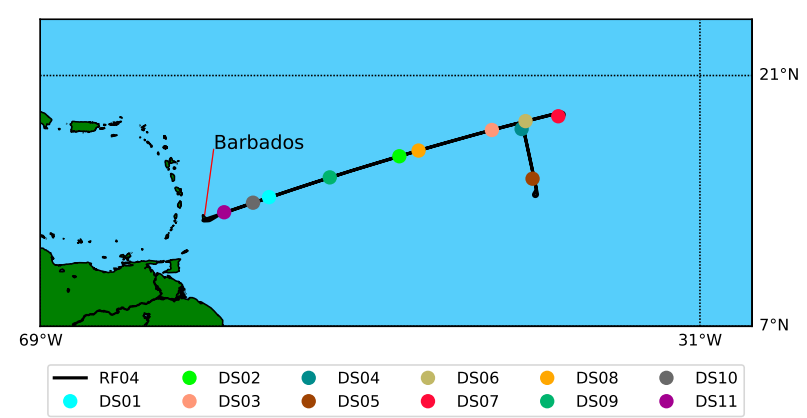

FIG. 1. The locations of the 11 dropsondes (circles) launched during NARVAL-South campaign research flight 4 (RF04) are shown in different colors that will be used in profiles in figures that contain more than one dropsonde per plot. RF04 traveled approximately $2000 \mathrm{~km}$ across the North Atlantic trade wind region before returning to Barbados. The basis for the figure is credited to the NARVAL campaign report by (Klepp et al. 2014). 

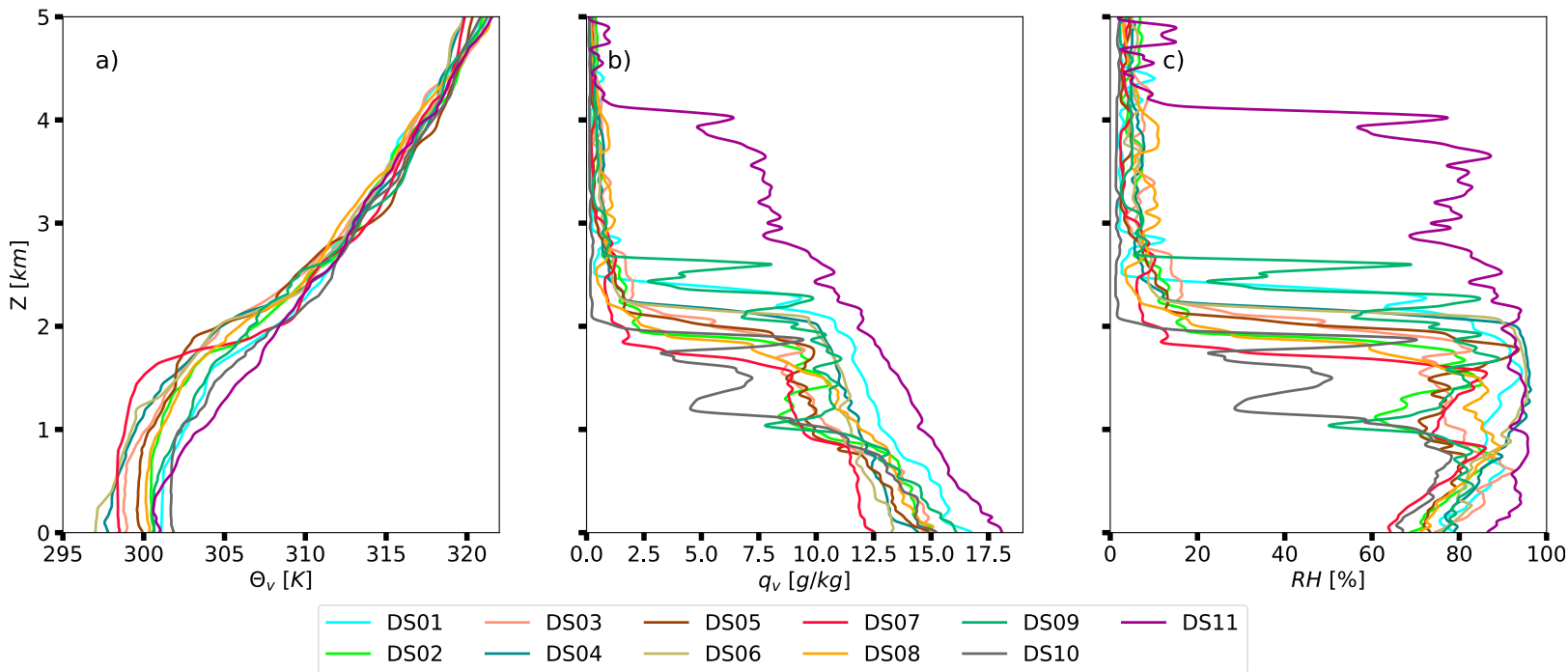

FIG. 2. Profiles for (a) virtual potential temperature $\Theta_{v}$, (b) specific humidity $q_{v}$, and (c) relative humidity (RH) with respect to altitude, recorded for each of the dropsondes launched during RF04.

DS launches. For the simulations in this study, DALES was run using the kappa scalar advection scheme recommended in Hundsdorfer et al. (1995) and also described in Heus et al. (2010). DALES can be run with either a two-moment microphysics scheme that is based on the Seifert Beheng scheme (Seifert and Beheng 2006) or a scheme by Khairoutdinov and Kogan (2000). Similarly, there are two main subgrid-scale schemes in DALES, the subfilter-scale turbulence kinetic energy scheme and a Smagorinsky subfilter scheme. Downward radiation at the top of the model is calculated using the GCM profiles above the model ceiling.

DALES has been successfully used to simulate trade wind cumulus for scientific research, and has shown satisfactory skill in reproducing key features of this cloud regime based on various intercomparison studies such as BOMEX (Siebesma et al. 2003), RICO (vanZanten et al. 2011), Atlantic Trade Wind Experiment (ATEX; Stevens et al. 2001), transition cases (van der Dussen et al. 2013; Neggers et al. 2017), and continental cumulus (Brown et al. 2002). The DALES code allows continuous nudging of liquid water potential temperature $\Theta_{l}$, the total water mixing ratio $q_{t}$, and $u$ and $v$ winds throughout the simulation. For a detailed description of the DALES model, including details on the schemes and processes included in the model we refer to Heus et al. (2010). (DALES is an open access code and is available for download from the DALES team on GitHub at https://github.com/dalesteam.)

\section{c. GCM dataset}

The method of using GCM data from ECMWF to derive the large-scale forcings for DALES has been previously used by Dal Gesso and Neggers (2018) and more recently by van Laar et al. (2019). We refer to the latter study for a detailed description of the forcing data and method. These large-scale forcings, from IFS weather analyses, are based on an average over a $0.5^{\circ} \times$ $0.5^{\circ}$ domain centered over the DS location. The vertical resolution of the IFS dataset consists of 91 pressure levels with a high vertical resolution at the surface and a coarser resolution near the ceiling of the dataset. We combined 12-hourly data from weather analyses with short-range 3-hourly forecasts to fill up the time points in between. This yields a continuous forcings dataset at a temporal resolution of $3 \mathrm{~h}$. IFS data from ECMWF have also been used in various other recent LES studies for calculating the large-scale forcings (e.g., Gustafson et al. 2017).

To investigate the difference between the IFS dataset at the time of each DS launch, the ensemble-mean profile for $\Theta_{v}$ and $q_{v}$ across all IFS datasets is compared to the ensemble-mean DS profile across all DSs. Figure 3 shows the ensemble-mean $\Theta_{v}$ and $q_{v}$ profiles for all DSs and IFS profiles. The median value of both ensembles at each altitude is represented by the solid line while the shaded region indicates the area between the 5 th and 95 th percentiles. It should be noted that the median IFS $\Theta_{v}$ and $q_{v}$ profiles are colder and drier at the surface. This indicates that the IFS slightly underestimates the temperature and humidity in the lowest layers of the BL. While there is a slightly greater variability in the DS profiles near the surface, in general the IFS is able to capture the basic observed thermodynamic structure of the trade wind BL. 

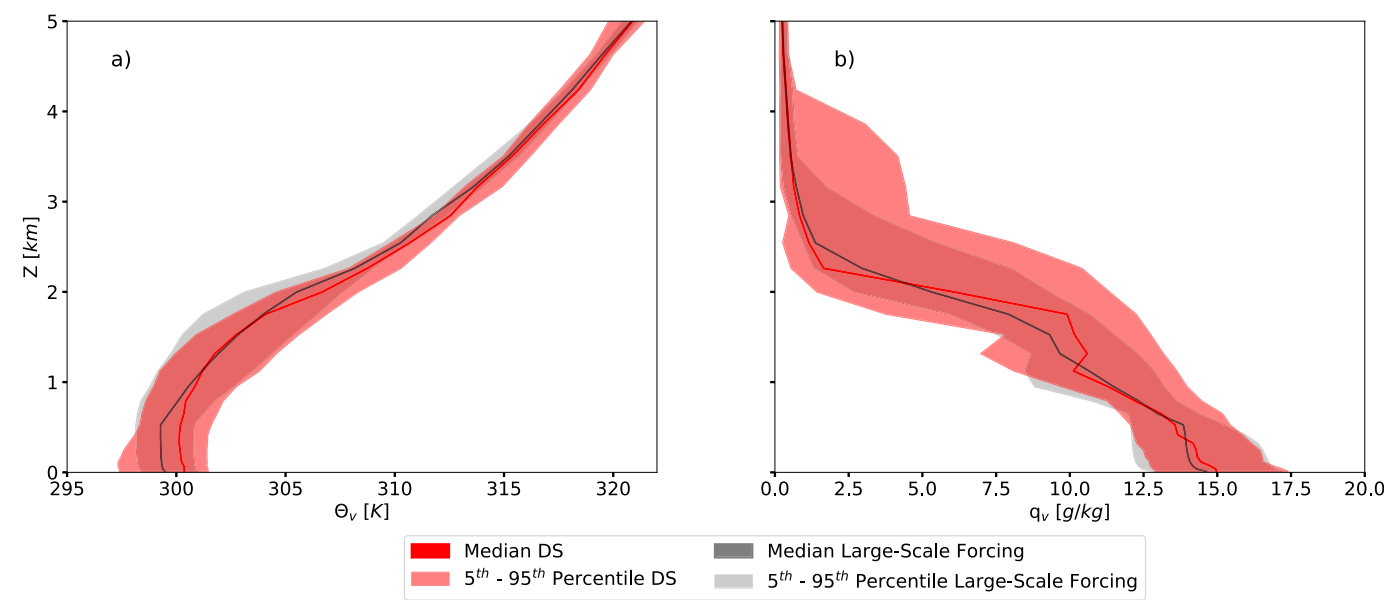

FIG. 3. The profiles of (a) virtual potential temperature $\Theta_{v}$ and (b) specific humidity $q_{v}$ for an ensemble of all the DS profiles (red) and all IFS data (gray) for the Sim_Con simulations. Solid lines represent the median profiles while the shaded region represents the area between the 5th and 95th percentiles of the ensembles.

To gain more insight into the atmospheric conditions represented in the IFS dataset, the profiles of pressure velocity $\omega$ are studied at the DS launch times, shown in Fig. 4. Figure 4a indicates that there is large variation in the magnitude and vertical structure of $\omega$ within the sampled area. From Fig. 4a a relatively linear change is seen in $\omega$ within the lowest $1 \mathrm{~km}$ of the atmosphere. Therefore, the value of $\omega$ at $1 \mathrm{~km}$ for each DS time is compared to the corresponding IFS $q_{v}$ value at $1 \mathrm{~km}$, shown in Fig. 4b. The large-scale convergence $(\omega<0)$, expressed by large-scale ascent, tends to be associated with higher low-level humidity, while largescale divergence $(\omega>0)$, as expressed by descent, is associated with drier conditions. The expected association between ascent and high humidity values, and between descent and drier conditions is confirmed by the linear fit line in Fig. 4b.

\section{Experimental configuration}

\section{a. General strategy}

The novel approach used in this study is to directly blend the DS profiles into the forcing data as derived from a GCM in order to nudge the LES toward observed profiles. Simulations are then generated at the location of each DS launch. The aim is to investigate and understand the subsequent impact on the thermodynamic state in the LES near the time point of the DS. There is a particular focus on the representation of the BL deep structure and the trade wind inversion. Methods to fairly and optimally compare the observed inversion as derived from the point-sampled DS profiles to that of the three-dimensional LES domain are explored. The associated impact on clouds will be briefly documented, but their detailed analysis and evaluation against HALO data are for now considered an ongoing research topic.

\section{b. DALES setup}

This study utilizes time-dependent large-scale forcing, including the application of prescribed advective tendencies and prescribed large-scale subsidence. Largescale horizontal advection is prescribed for all prognostic variables. The large-scale vertical advection tendency is interactive with model profiles by using prescribed subsidence. For wind, the geostrophic forcing is derived from the large-scale pressure field. The lower boundary conditions for these simulations include prescribed sea surface temperature (SST), roughness length, and interactive fluxes. The simulations are generated with an interactive radiation scheme and warm Seifert Beheng two moment bulk microphysics scheme (Seifert and Beheng 2006).

Continuous nudging is applied toward a reference state at a slow, synoptic time scale of $3 \mathrm{~h}$. The nudging in these simulations is applied in an effort to reduce the drift in the LES and involves nudging the simulation toward the large-scale forcing profiles in the IFS dataset. The nudging tendency toward the reference state is carried out using the following equation:

$$
\left.\frac{\Delta \bar{\phi}}{\Delta t}\right|_{\operatorname{nudged}(x, y, z)}=\frac{\bar{\phi}_{n}(z)-\bar{\phi}(z)}{\tau}
$$

where $\Delta t$ is the time between each of the large-scale forcings time steps, and $\bar{\phi}_{n}(z)$ is the reference profile toward which the LES profile $\bar{\phi}(z)$ is nudged. The nudging tendency is the same for every grid cell, with 


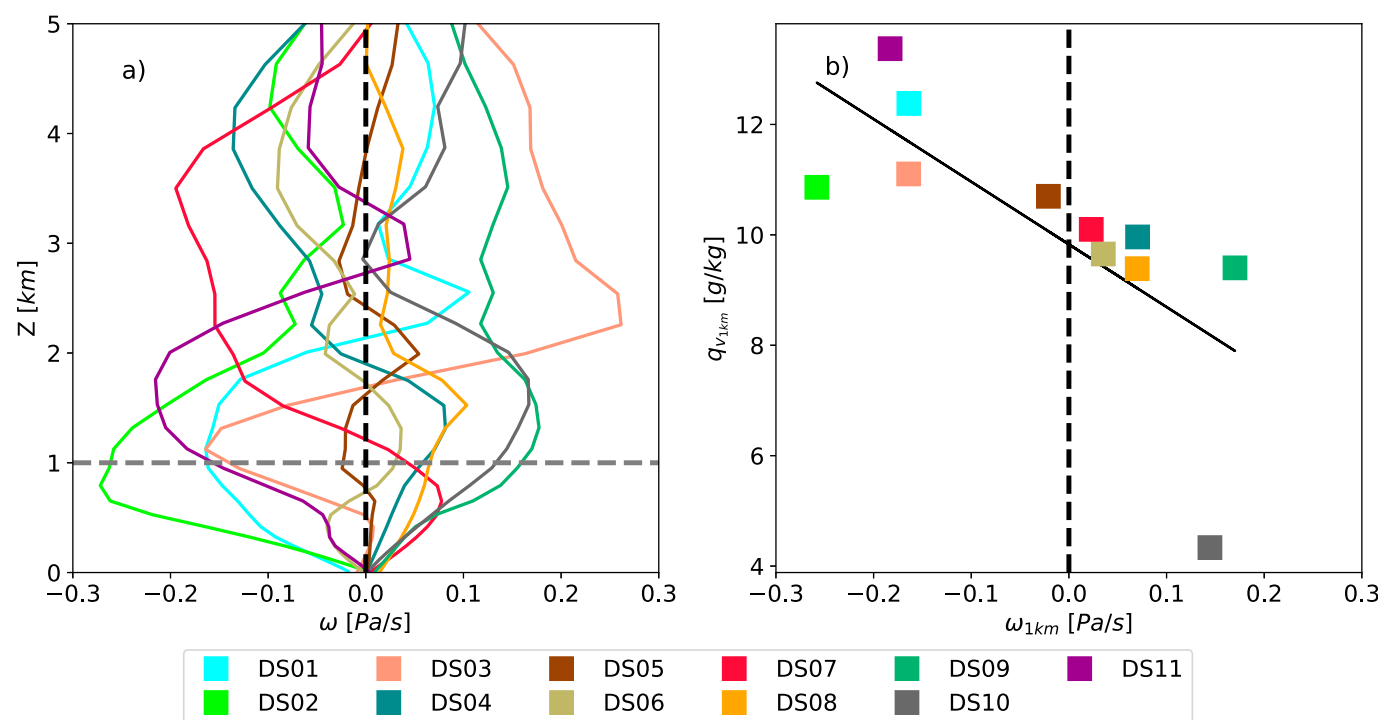

FIG. 4. The IFS pressure velocity $\omega$ (a) vertical profiles for each DS location and time and (b) value at $1 \mathrm{~km}$ compared to the corresponding IFS $q_{v}$ value at $1 \mathrm{~km}$.

$\bar{\phi}(z)$ linearly interpolated between the IFS time steps. The nudging time scale is represented as $\tau$, where large values of $\tau$ indicate weak nudging and small values of $\tau$ indicate tight nudging. The value of $\tau$ in this study is set to $3 \mathrm{~h}$, which allows the simulation to take the large-scale data into account while also allowing the turbulence in the simulation to act freely. This method was demonstrated by Neggers et al. (2012) at the location of a permanent supersite, and more recently by van Laar et al. (2019).

The simulations are generated on a domain that was fixed in space (Eulerian) at each DS location. The domain size used in this study is $12.8 \mathrm{~km} \times 12.8 \mathrm{~km} \times$ $5.04 \mathrm{~km}$ with a resolution of $50 \mathrm{~km} \times 50 \mathrm{~km} \times 40 \mathrm{~m}$. The general details of resolution and nudging time scale for the two main sets of simulations are summarized in Table 2.

\section{c. Overview of simulations}

\section{1) Control simulations}

A set of 11 control simulations were generated, referred to as Sim_Con, and were nudged toward the pure IFS state. The time series of the pure IFS state is visualized in Fig. 5a as a timeline of atmospheric states where the blue circles indicate an individual time step $t$ in the IFS dataset. Each time step $t$, in Fig. 5, contains the largescale forcing profiles required by the LES. The subscripts $k$ represent where each specific $t$ is in relation to the time of the DS launch, where $t_{k}$ is the time step directly before the time point of the DS launch. The time difference between each $t$ is exactly $3 \mathrm{~h}$.
Each Sim_Con was initialized at 0000 UTC and run for $24 \mathrm{~h}$. The nudging is implemented in the Sim_Con cases in order to reduce as many differences as possible between the different simulations carried out in this study.

\section{2) DROPSONDE SIMULATIONS}

The second tier of 11 simulations, referred to as Sim_DS, has a similar setup to the Sim_Con. The main difference between the setup of Sim_DS and Sim_Con is that the DS profile is inserted into the IFS dataset at the time point of the DS launch. To effectively blend the DS profile into the IFS nudging dataset the following procedure, made up of three steps, is designed.

First, at the exact time point of the DS launch, a new time step is created in the IFS time series, $t_{\mathrm{DS}}$. This time step is indicated by the green square in Fig. 5b. To calculate the radiation, the DS profiles are used below the flight altitude of HALO while the IFS profiles are used above this altitude. The second step is then to define two new time steps, $3 \mathrm{~h}$ before and after $t_{\mathrm{DS}}$. This creates a nudging time window $\left(T=t_{a 1}-t_{a 0}\right)$ of $6 \mathrm{~h}$. At these

TABLE 2. Setup of simulations generated. The simulations analyzed in the initial experiments are highlighted in boldface.

\begin{tabular}{llrrrrrr}
\hline \hline Parameter & Simulation & \multicolumn{7}{c}{ Value } \\
\hline$\tau(\mathrm{h})$ & Sim_Con & 1 & 2 & $\mathbf{3}$ & 4 & 5 & 6 \\
& Sim_DS & 1 & 2 & $\mathbf{3}$ & 4 & 5 & 6 \\
$T(\mathrm{~h})$ & Sim_DS & 3 & $\mathbf{6}$ & 12 & - & - & - \\
$\Delta z(\mathrm{~m})$ & Sim_DS & 10 & 20 & $\mathbf{4 0}$ & - & - & - \\
\hline
\end{tabular}



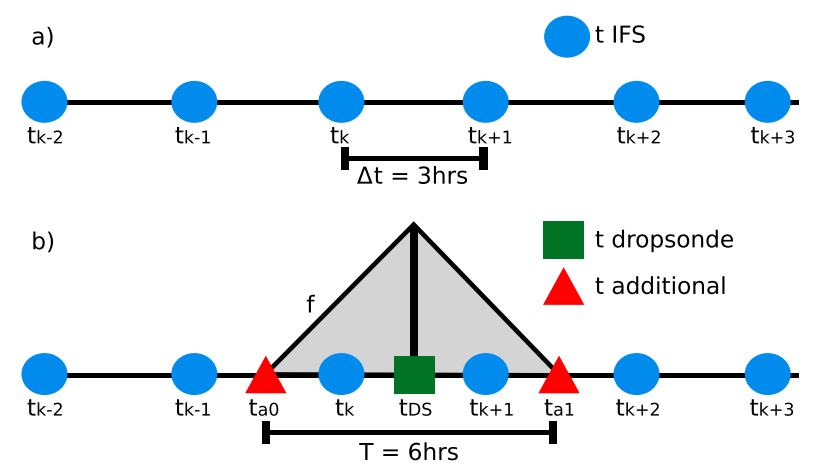

FIG. 5. (a) Schematic of the time series of the IFS, from ECMWF, with each time step $t$ (blue circles) contains the largescale atmospheric profiles at that location and time of day for Sim_Con. (b) As in (a), but for Sim_DS and also includes the DS profiles, on the IFS vertical grid, at the DS launch time (green square; $t_{\mathrm{DS}}$ ) and two additional time steps (red triangles; $t_{a 0}$ and $t_{a 1}$, which are located, respectively, $3 \mathrm{~h}$ before and after the DS launch time). These two additional time steps allow for a gradual nudging toward the DS profile, and are created by linearly interpolating the atmospheric profiles from the adjacent IFS time steps.

two new time steps, represented by the red triangles in Fig. 5b, the values from the atmospheric profiles at the two adjacent IFS time steps are linearly interpolated onto $t_{a 0}$ and $t_{a 1}$.

The final step is then to adjust each of the profiles at time steps $t_{k}$ and $t_{k+1}$. The closer time step $t_{k}$ or $t_{k+1}$ is to $t_{\mathrm{DS}}$, the more it resembles the DS state. This is achieved by using a gradual changing weight $f$ :

$$
\begin{gathered}
f=\left\{\begin{array}{l}
\left|\frac{t_{a 0}-t_{k}}{t_{a 0}-t_{\mathrm{DS}}}\right| \text { for } t_{k}<t_{\mathrm{DS}} \\
\left|\frac{t_{a 1}-t_{k}}{t_{a 1}-t_{\mathrm{DS}}}\right| \text { for } t_{k} \geq t_{\mathrm{DS}}
\end{array}\right. \\
\bar{\phi}_{t_{k}}^{\star}=\bar{\phi}\left(t_{k}\right)(1-f)+\bar{\phi}\left(t_{\mathrm{DS}}\right) f,
\end{gathered}
$$

where $\bar{\phi}\left(t_{\mathrm{DS}}\right)$ is the DS profile on the IFS vertical grid as a domain-average profile, and $\bar{\phi}\left(t_{k}\right)$ is original domain-average profile at time step $t_{k}$. As illustrated by Fig. 5b, to adjust the profile at the time step $t_{k}$, situated directly before the $t_{\mathrm{DS}}, f$ is calculated using Eq. (2). Using $f$, the new profile $\bar{\phi}_{t}^{\star}$ at time step $t_{k}$ is calculated using Eq. (3).

Summarizing, the new time series contains three new time steps, together defining a nudging time window in which the reference state for nudging is modified to reflect the atmospheric state observed by the DS. The addition of the new time steps $t_{a 0}$ and $t_{a 1}$ in the sequence ensures that the impact of the DS is not felt before the simulation enters the time window.
This also ensures that the differences in the proximity of $t_{\mathrm{DS}}$ to the adjacent IFS time steps do not affect the nudging.

\section{Model results}

\section{a. Results for all Sim_Con cases}

Previous studies, such as Nuijens et al. (2014) at the BCO and Lamer et al. (2015) at the ENA site, have reported observations of strong temporal evolutions of the BL in the trade wind region. To assess if this is also seen in simulations for the NARVAL campaign, the time-height contour plots of $\mathrm{RH}$, including the evolution of the cloud layer, for all 11 Sim_Con are shown in Fig. 6. All simulations contain clouds but differ considerably. Indeed, all simulations show time variation on a short time scale. We speculate that this variation is introduced by time-varying forcings. The cloud base $Z_{\mathrm{CB}}$ is defined as the lowest level at which the cloud fraction $(\mathrm{CF})$ is greater than $0.001 \%$, while cloud top $Z_{\mathrm{CT}}$ is defined as the level above which the $\mathrm{CF}$ is less than $0.001 \%$. The results from Fig. 6 indicate that (i) the LES is able to reproduce the strong temporal evolution of the BL, and (ii) this behavior that Nuijens et al. (2014) observed is not unique to the BCO.

As the simulations in Sim_DS are directly forced toward the DS profiles, it is beneficial to compare the simulations to an independent set of measurements to investigate their representativeness of local conditions. To this purpose, a time series of IWV was retrieved by HAMP. The median IWV retrieval, calculated over a 1-h window around each DS launch time, is compared to the IWV for the domain-averaged profile at $t_{\mathrm{DS}}$. From Fig. 7 it is shown that the LES is able to capture IWV values similar to the observed values. This comparison indicates that the LES is capable of representing the atmospheric conditions observed over the flight path. It should be noted that DS11 is not included in this comparison as the aircraft landed less than half an hour after DS11 was launched and therefore there is not enough data to have a consistent comparison.

\section{b. DS01 results}

To understand the impact of nudging the LES toward the DS, in particular on the BL and its clouds, we focus on a single experiment. This experiment corresponds to the temporal and spatial region of the first dropsonde, DS01. First, a visual indication for both simulations is shown in Fig. 8. These visualizations give an instantaneous look at the impact of including the DS profile into the LES setup. To quantify the difference, the CF and liquid water path (LWP) for the domain at that time 

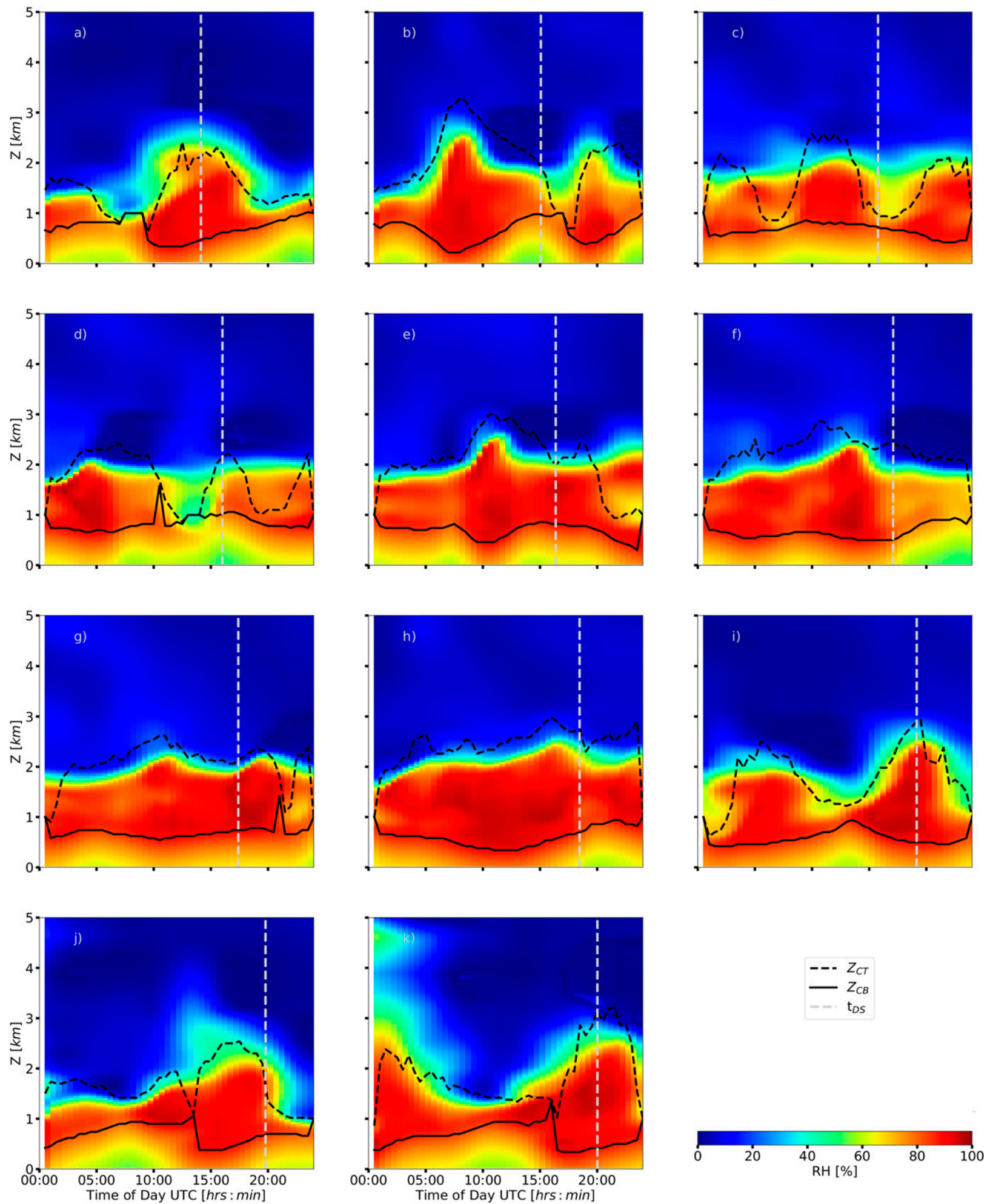

FIG. 6. Time-height contour plots of the relative humidity (RH) of the Sim_Con for the domains centered over each DS, representing the locations from (a) DS01 to (k) DS11. The time at which each DS is launched is represented by the gray dashed lines, while time series of the altitudes of the cloud top $Z_{\mathrm{CT}}$ and cloud base $Z_{\mathrm{CB}}$ are shown by the dashed black lines and solid black lines, respectively. 
point were calculated. There is a difference of $21.8 \%$ in $\mathrm{CF}$ and of $0.03 \mathrm{~kg} \mathrm{~m}^{-2}$ in LWP between the simulations.

One aim of this study is to investigate the impact on the BL structure by nudging the LES toward the DS profile. To this purpose the DS profiles for potential temperature $\Theta, q_{v}$, and $\mathrm{RH}$ are compared to the domainaveraged LES profiles at $t_{\mathrm{DS}}$. The comparison of LES profiles, shown in Fig. 9, indicate that a number of features in the atmosphere are captured well by both the Sim_Con and Sim_DS01. These features include the inversion height, the mixing-layer height and state, and the lapse rate in the free troposphere. It should be noted that the inversionlayer depth and strength should not be directly compared between a domain-averaged profile and a point-sampled profile. A statistical method for comparing inversion height and strength to deal with this problem is explored in a more detailed way in sections $4 \mathrm{~d}$ and $4 \mathrm{e}$.

The details of Fig. 9 show that the LES reproduces the observed vertical structure of the cumulus-capped $\mathrm{BL}$ to a reasonable degree. This result is commensurate with most previous LES studies of shallow cumulus in the trades (Siebesma et al. 2003; vanZanten et al. 2011). In Fig. 9a the region that shows the largest effect of the nudging toward the DS is in the layer between approximately 2 and $3 \mathrm{~km}$. This is in the region of the inversion layer. It should be noted though that this difference is still quite small. The effect of nudging the simulation toward the DS is more pronounced for variables $q_{v}, \mathrm{RH}$, and CF. The most significant difference between the Sim_Con and Sim_DS01 profiles occurs in Fig. 9d where the vertical profile of CF is changed as a result of nudging the simulation toward the DS. Compared to the single peak at cloud base in the Sim_Con simulation, the Sim_DS01 profile consists of two peaks, one at cloud base and one at cloud top. The second peak in the $\mathrm{CF}$ is potentially related to outflow clouds just below the inversion layer. This is also seen in the $3 \mathrm{D}$ visualization in Figs. $8 \mathrm{c}$ and $8 \mathrm{~d}$.

Due to the importance of wind speed (Nuijens and Stevens 2012) and as the profiles can be quite variable throughout the troposphere, particularly in the boundary layer, it is beneficial to determine how well the LES can represent the wind speed profiles compared to the observations. To this purpose the wind speed profiles, $u$ and $v$, are shown in Figs. 9e and 9f. The profiles shown for DS01 are based on recordings of wind speed by the GPS system on board the DS. Both Sim_Con and Sim_DS01 show a good ability to capture the general profile. However, both simulations tend to underestimate the sharp changes in wind speed throughout the profile. Based on the profiles, it is indicated that the LES can represent the atmospheric conditions to a reasonable degree.

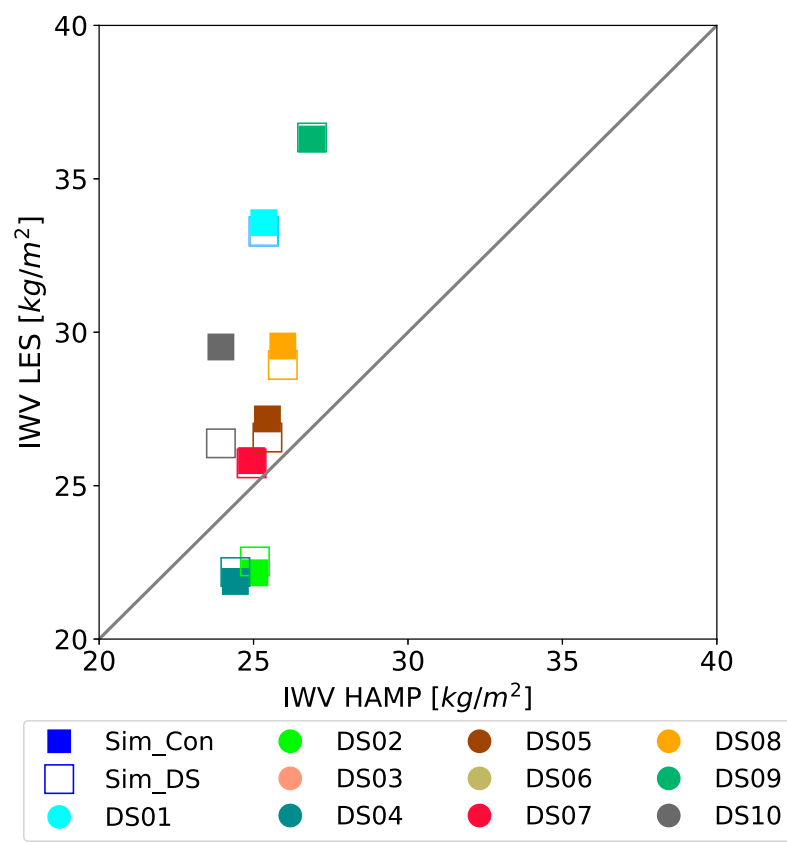

FIG. 7. Comparison between the IWV calculated from the Sim_Con (unfilled squares) and Sim_DS (filled squares) for each DS location and the median IWV value from a 1-h window of HAMP retrievals around the DS launch time.

We next focus on a visual comparison of the trade wind inversion in the water vapor profiles. In the case of both simulations the inversion occurs at approximately $2.4 \mathrm{~km}$. In general, domain-averaged LES profile of $q_{v}$ exhibit slight differences between Sim_Con and Sim_DS01, with the latter showing a slightly sharper change in the profile at the capping inversion height. Although the nudging toward the DS profile does appear to increase the strength of the inversion, both simulations seem to underestimate the inversion strength. One suspected cause of this underestimation is that the LES profiles being compared are domain averages. A more detailed comparison of the inversion height and strength are further investigated in sections $4 \mathrm{~d}$ and $4 \mathrm{e}$, respectively.

To quantify the difference between Sim_Con and Sim_DS01 in the domain-averaged profiles, the differences between the profiles of $\mathrm{CF}$ and $q_{v}$ were calculated for every time step in the LES. The resulting timeheight contour plots are shown for $\mathrm{CF}$ and $q_{v}$ in Figs. 10a and $10 \mathrm{~b}$, respectively. As expected, before the nudging time window $T$ begins, there are no differences between the simulations for both variables. The differences in the simulations begin slowly after the start of $T$ and gradually increase in strength, particularly around $t_{\mathrm{DS}}$. Most of the differences in the simulations tend to occur within the cloud region, with a larger CF in Sim_DS01 compared to Sim_Con at around $2 \mathrm{~km}$. This is also true 


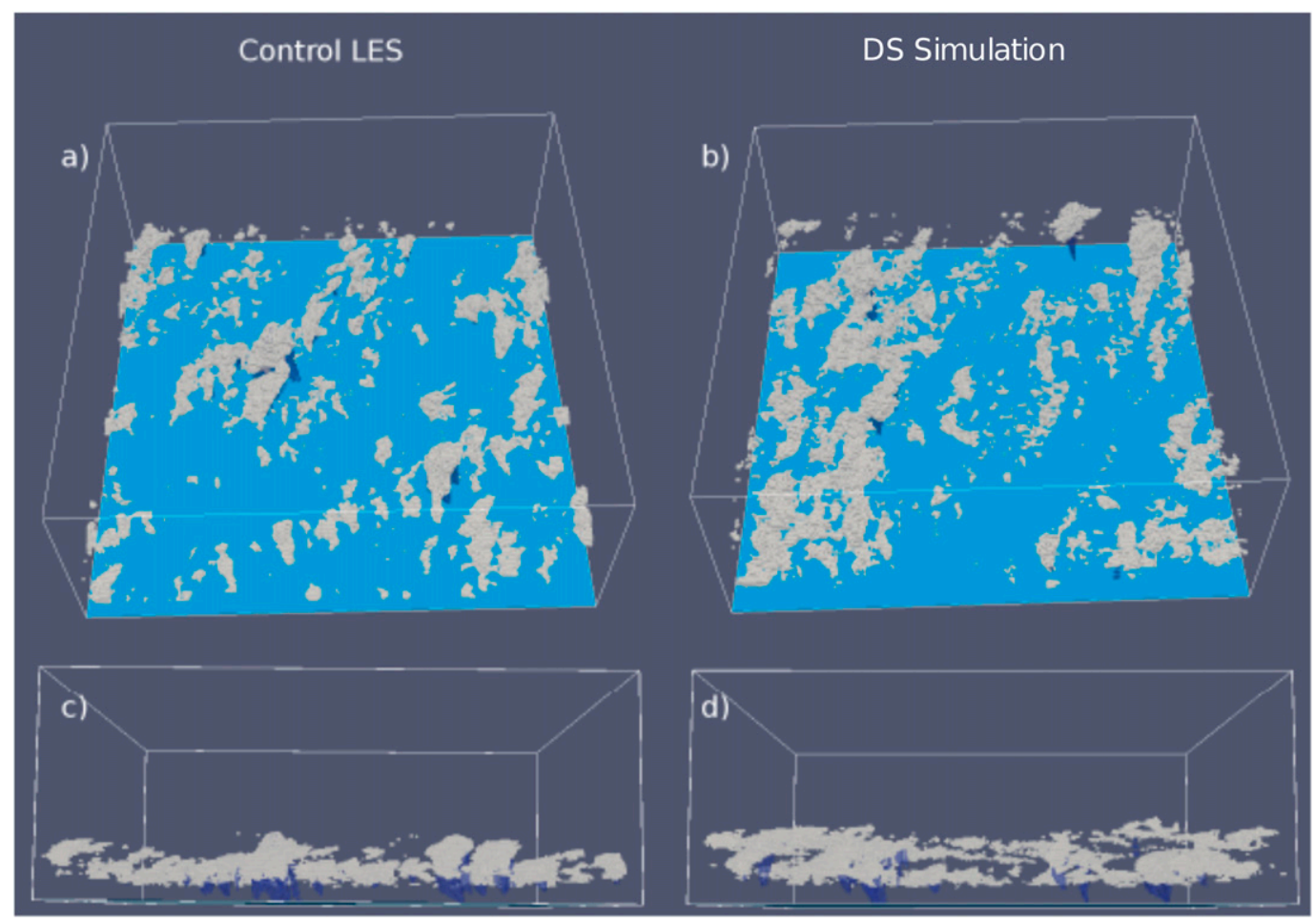

FIG. 8. A 3D visualization of simulated clouds and precipitation at 1400 UTC 14 Dec 2013. The light blue shows the ocean surface, the light gray shows the surface of the clouds, and the dark blue indicates precipitation. (a),(b) Tilted top views and (c),(d) side views of the cloud fields for (left) Sim_Con and (right) Sim_DS01.

for the difference in $q_{v}$. The strengthening of the inversion is highlighted by the larger values of $q_{v}$ at lower altitudes in Sim_DS01, compared to the corresponding values in the Sim_Con, and lower values of $q_{v}$ at higher altitudes. The time-height contour plot also indicates that the impact of including the DS data in the forcing lasts for a long period after $T$ ends.

\section{c. Dropsonde impact analysis}

We now progress from a single case to assessing the impacts for all 22 simulations. To this purpose, a special metric is adopted to express the deviation of LES from the observations concerning the vertical structure within the BL. To quantify the impact of including the DS data on the thermodynamic state of the BL, the areas between the domain-averaged LES profiles and the corresponding observed DS profiles were calculated. It should be noted that the DS profiles have been linearly interpolated onto the LES vertical grid to ensure a fair calculation of the area between the profiles. The time evolution of these areas during $T$ is then assessed for all 22 cases.

Suppose $A_{\phi}$ is the area enclosed between the simulated and observed profiles of variable $\phi$ in the lowest $3 \mathrm{~km}$ :

$$
A_{\phi}=\int_{0 \mathrm{~km}}^{3 \mathrm{~km}}\left|\bar{\phi}_{\mathrm{LES}}(z)-\phi_{\mathrm{DS}}(z)\right| d z
$$

where $\phi \in q_{v}, \Theta, \mathrm{RH}$, or any variable of interest, with LES and DS indicating the domain-averaged and observed profiles, respectively. This area is calculated as a function of time during $T$ and is done for both the Sim_Con and Sim_DS simulations. Time is synchronized between all simulations such that $t=0$ at $t_{\mathrm{DS}}$. For Sim_Con, the area will be referred to as $A_{\phi, \text { Sim_Con, }}$ while for Sim_DS it is $A_{\phi, S i m \_D s}$. The next step is to normalize these areas with respect to $A_{\phi, S i m}$ Con $a t t_{\mathrm{DS}}$. The normalization yields the following two nondimensional expressions for the skill $S$ of the simulation in matching the DS profile:

$$
\begin{aligned}
S_{\phi, \text { Sim_Con }}(t) & =\frac{A_{\phi, \text { Sim_Con }}(t)}{A_{\phi, \text { Sim_Con }}(t=0)}, \\
S_{\phi, \text { Sim_DS }}(t) & =\frac{A_{\phi, \text { Sim_DS }}(t)}{A_{\phi, \text { Sim_Con }}(t=0)} .
\end{aligned}
$$

Note that the division of both metrics by $A_{\phi, \text { Sim }}$ Con $(t=0)$ makes these quantities directly comparable. It should also be noted that the lower the value of $S_{\phi}(t)$ 

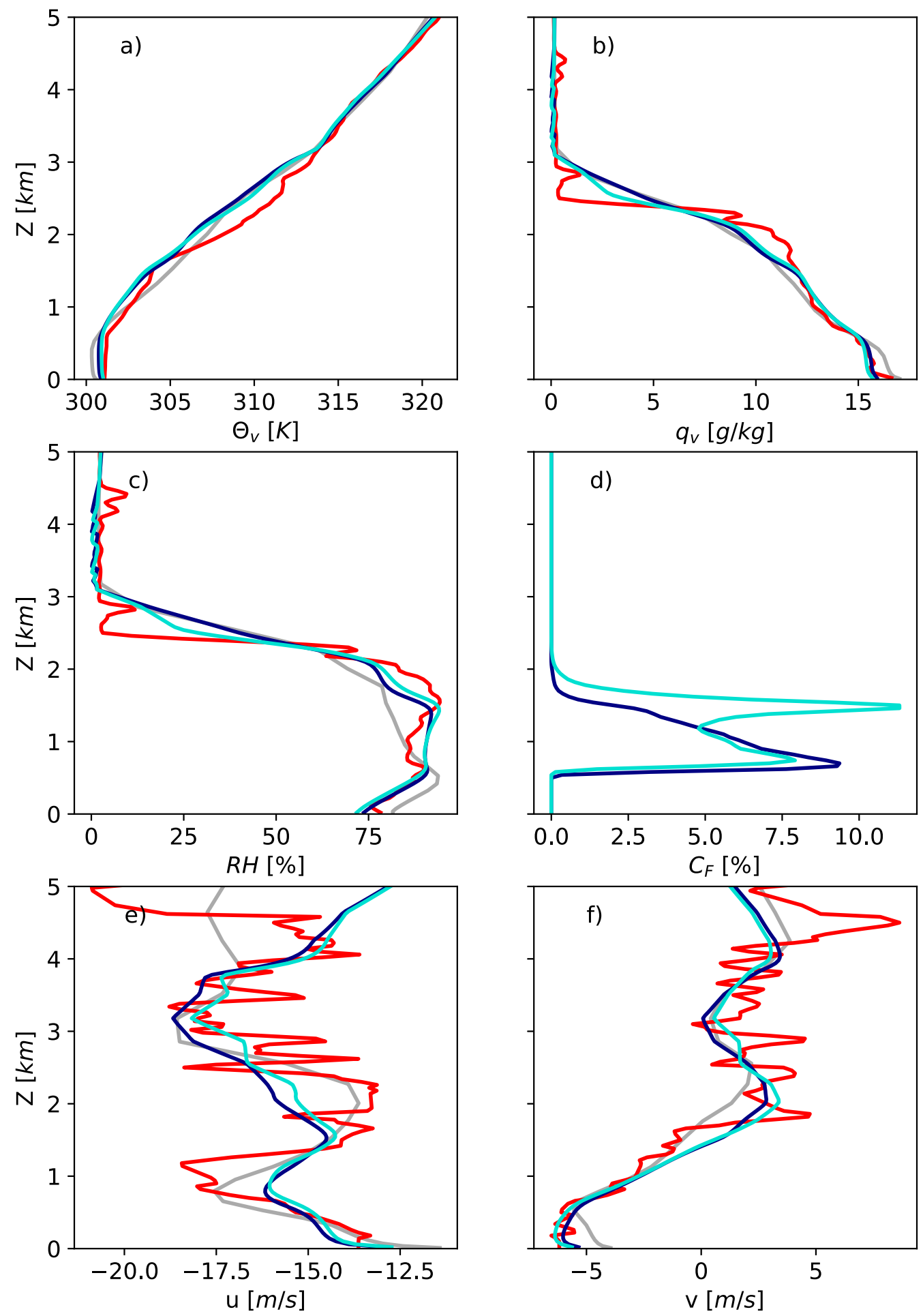

IFS Sim_Con

DS01

Sim_Con

Sim_DS01

FIG. 9. Atmospheric profiles of potential temperature $\Theta$, water vapor specific humidity $q_{v}$, relative humidity (RH), cloud fraction (CF), west-east wind speed velocity $u$, and south-north wind speed velocity $v$ for DS Observations (red), Sim_Con (navy), and Sim_DS01 (light blue). The IFS for the control simulations (IFS_Con; gray) for the time when DS01 was launched are included for reference. 

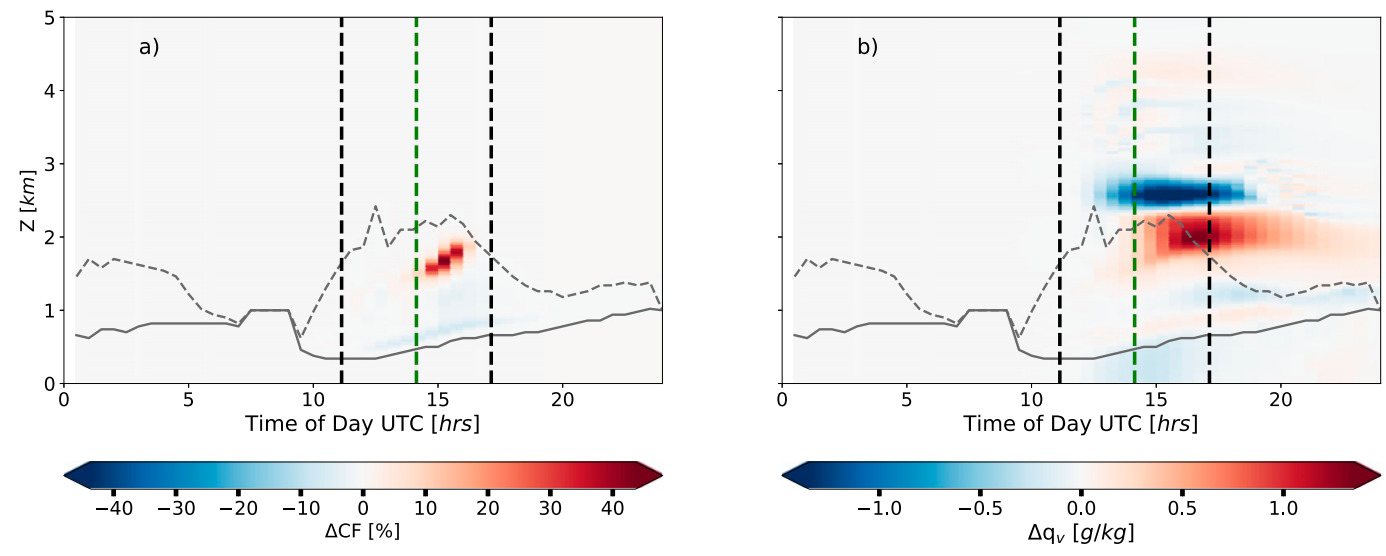

$$
---Z_{C T} \quad-Z_{C B} \quad--\cdot t_{D S} \quad--\cdot \text { Nudging Window (T) }
$$

FIG. 10. Time-height contour plots of the difference between Sim_DS01 and the Sim_Con for (a) CF and (b) $q_{v}$. $\Delta \mathrm{CF}$ and $\Delta q_{v}$ are greater than 0 if the value for Sim_DS01 is greater than the corresponding value in the Sim_Con. The nudging time window is shown by the dashed black lines while the DS launch time is shown by the dashed green line. The times series for cloud-top and cloud-base altitudes are shown for Sim_Con as the dashed and solid gray lines, respectively, as in Fig. 6a.

the better the simulation is at representing the DS profile.

To see if nudging the LES toward the DS improves the representativity of the domain-averaged profiles, this calculation is carried out for the profiles of three variables, $\Theta, q_{v}$, and $\mathrm{RH}$. A time series for the duration of $T$ is shown for each of these variables in Fig. 11. The shaded region in the time series designating the 5th95th percentiles, and the median indicated separately. Per definition $S=1$ for Sim_Con at $t_{\mathrm{DS}}$. In the case of all three variables the ensemble spread for the Sim_Con and Sim_DS are identical at the start of the time window, $t_{a 0}$. This reflects that until that point in time the forcing is identical. Following the beginning of the time window, the median value of $S$ for Sim_DS in all variables remains smaller than that of the Sim_Con, for a longer time period.

As expected, $S$ begins to decrease, in each of the variables, as Sim_DS begins to feel the effects of the DS data to a larger extent. Following $t_{\mathrm{DS}}$, Sim_DS feels the effects of the DS data to a lesser extent as time progresses. Therefore, the spread in the values of $S$ begins to increase again toward the end of $T$, as the simulations begin to feel the impact of the pure IFS state. The behavior of the time series for all three variables indicates that by including the DS profiles into the IFS data leads to better representation of the BL deep vertical structure.

\section{d. Inversion height}

Previous studies have shown that the strength of the trade wind inversion has a significant impact on the presence of clouds in the BL (e.g., Klein and Hartmann
1993). As a result of this and based on the visual impact of nudging in the region of the capping inversion in Fig. 9 it is important to determine if the capping inversions in the LES are representative of observations. To assess whether the visual impact of nudging leads to an improvement in the representativeness, a comparison of the inversion height and strength needs to be derived from both model data and observations. In literature, there have been a large variety of methods proposed for this (Albrecht et al. 1979; Stull 1988; Wood and Bretherton 2004; Teixeira et al. 2008; Lock 2009; Neggers 2015; Gentine et al. 2015).

This study explores the use of a new probabilistic method for determining the inversion height and strength. This method makes use of probability density functions (PDFs) of inversion height and strengths as diagnosed over all columns in the LES. It is designed to achieve a fair comparison between measurements and high-resolution simulations.

The first step is to calculate the discretized vertical gradient $\Gamma_{\phi}$ of a variable $\phi$ as follows:

$$
\Gamma_{\phi}=\left|\frac{\Delta \phi}{\Delta z}\right|,
$$

where $\phi \in\left\{q_{v}, \Theta_{v}\right\}$ and $\Delta$ represents the difference between two model levels. The next step is to apply this procedure to (i) the DS profile and (ii) all columns in the three-dimensional LES field at $t_{\mathrm{DS}}$. This yields a single inversion height for the DS and a PDF of inversion heights for the LES. The final step is to compare up to three modes, defined as the three 

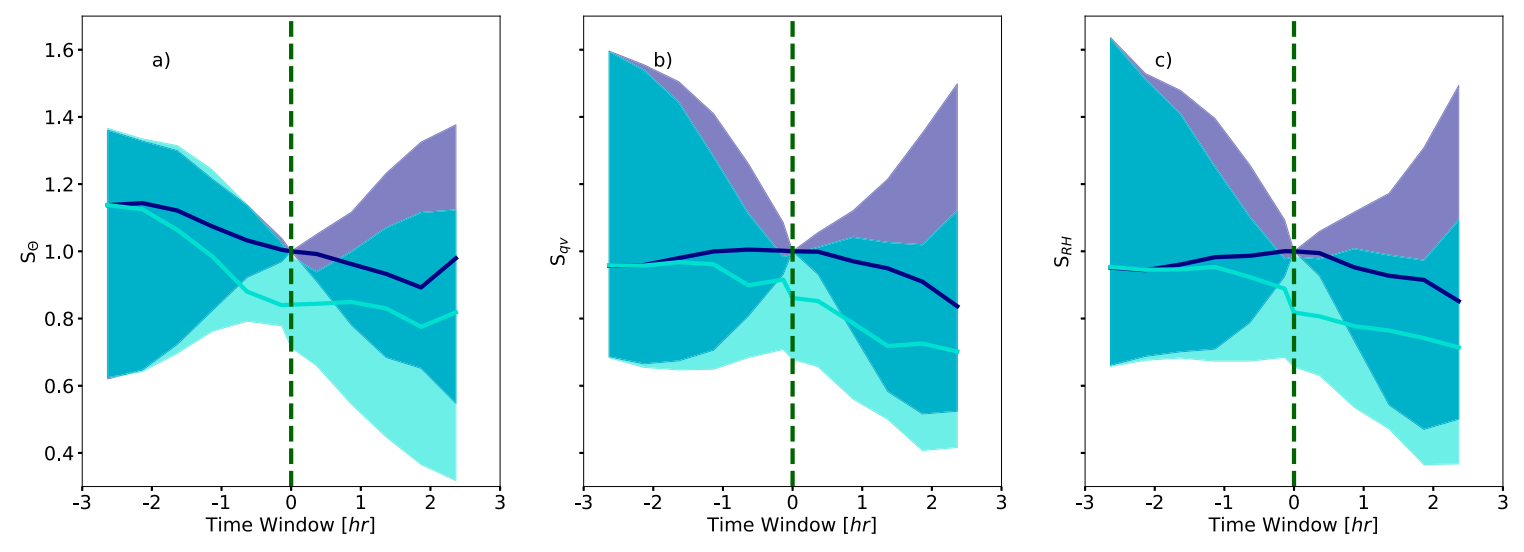

Sim_Con $50^{\text {th }}$ Percentile $\quad$ Sim_DS $50^{\text {th }}$ Percentile

- DS Launch Time

Sim_Con $5^{\text {th }} \rightarrow 95^{\text {th }}$ Percentile

Sim_DS $5^{\text {th }} \rightarrow>95^{\text {th }}$ Percentile

FIG. 11. Time series of the area between the domain-averaged profiles and the corresponding DS profile for (a) $\Theta$, (b) $q_{v}$, and (c) RH. The plots are centered on zero, the time of the DS launch (green dashed line), and takes into account the 6-h window during which Sim_DS is nudged toward the DS profile. The solid navy and light blue lines are the median time series of the skill for all 11 Sim_Con and 11 Sim_DS, respectively, while the shaded regions represent the 5th-95th percentiles across all time series.

largest local maxima in the PDF, to the single observed value. The closest mode is then selected as the inversion height of the LES, referred to as the selected mode.

A first benefit of using the probabilistic method described above is that it avoids comparing the domainaveraged profiles to single point samples. Second, by scanning multiple modes to establish the simulated inversion height we increase the chance that we select one that best reflects the physical meaning of the height that is derived from the DS. For example, at cloud base the vertical profiles can exhibit strong jumps, that are associated with the cumulus transition layer (Albrecht et al. 1995; Neggers et al. 2007). Also, the DS-derived height does not necessarily reflect the trade inversion. By including up to three modes it accounts for this possibility. The strongest gradients are typically seen at (i) the trade inversion, (ii) the cloud-base transition layer, and (iii) possibly one in between. By considering up to three modes instead of only one, the most relevant height is automatically considered. In the LES, the depth of the inversion layer reflects the spread among inversion heights over all columns in the grid, each calculated as the altitude of the strongest gradient. This means that the width of the PDF represents the depth of the inversion layer.

The probabilistic procedure is now illustrated with some concrete examples. Figures 12a and 12c show the observed and simulated inversion heights for DS01 and DS03, respectively. The first three LES modes are indicated for both Sim_Con (navy) and Sim_DS (light blue). The DS-derived inversion height is indicated by the dashed green line, while the LES selected mode is indicated by the crosses. Figures $12 \mathrm{~b}$ and $12 \mathrm{~d}$ also show the respective observed and domain-averaged $q_{v}$ profiles for reference. What is clear from these two examples is that the first three modes in LES reflect the trade inversion, the cloud-base inversion, and an additional third jump in the cloud layer. Interestingly, the trade inversion does not always have the largest mode in the LES. However, selecting the LES mode closest to the observed one still ensures that the most relevant inversion height in the LES is chosen for the comparison and also occurs relatively frequently in the domain.

To determine whether the inversion heights in the LES are representative of the DS-derived inversion heights, the selected modes in the inversion height PDFs are compared to the DS-derived inversion heights for all cases. Figure 13 shows the comparison between the altitudes of the selected modes, in Sim_Con and Sim_DS, compared to the inversion heights in the corresponding DS profiles, for (a) $\Theta_{v}$ and (b) $q_{v}$. It should be noted that all 11 Sim_DS are included in Fig. 13a; however, the selected mode for two of the Sim_DS cases occurs at the same altitude as the selected modes for two of the other Sim_DS cases. From this comparison it is therefore concluded that the LES is successful in capturing inversions at altitudes that are observed by the DSs for both $\Theta_{v}$ and $q_{v}$.

\section{e. Inversion strength}

Next the inversion strength is calculated. To this purpose, only the grid locations that have their inversion height at the level of the selected mode are considered. The median over the subset of inversion strengths is then compared to the DS-derived inversion strength. An example of the inversion strength PDFs derived for Sim_Con and Sim_DS01 is described in the appendix.

Figure 14a shows that both the Sim_Con and Sim_DS reproduce the observed DS inversion strength in $\Theta_{v}$ to a reasonable degree. However, the spread among 

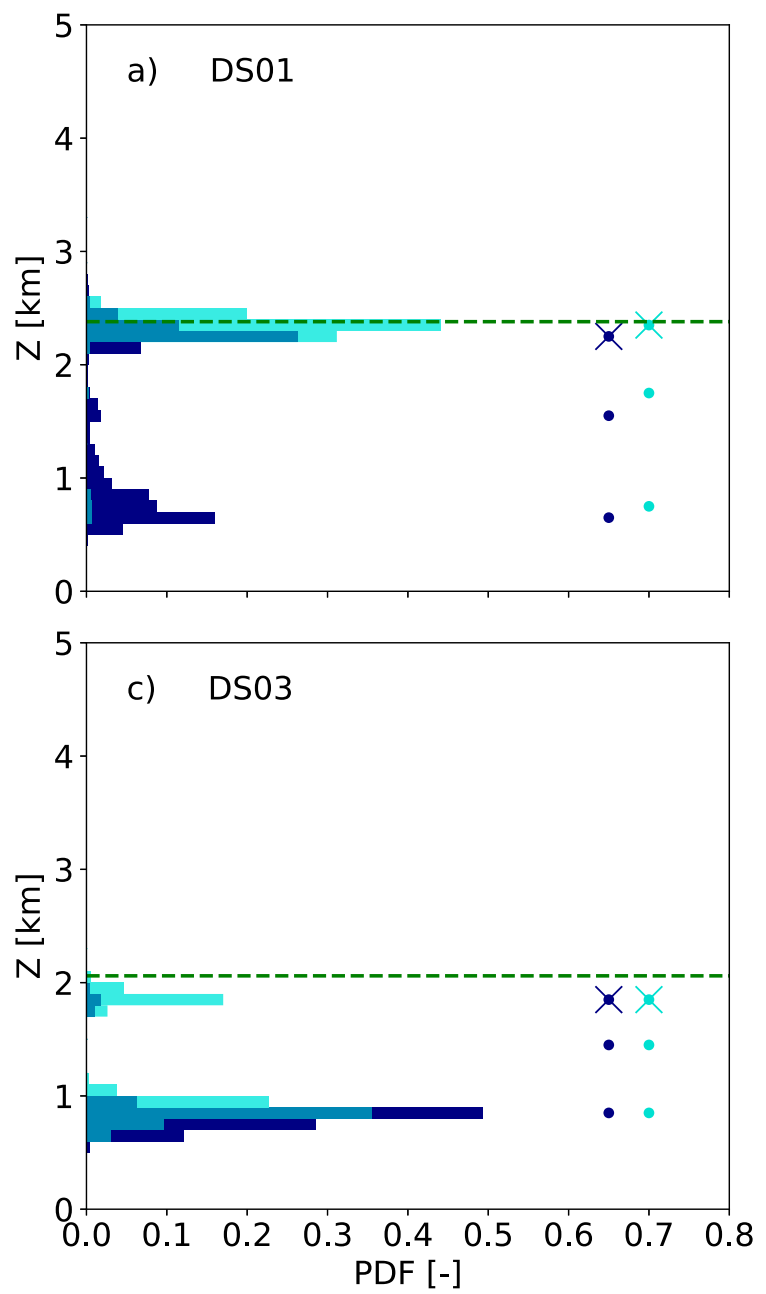
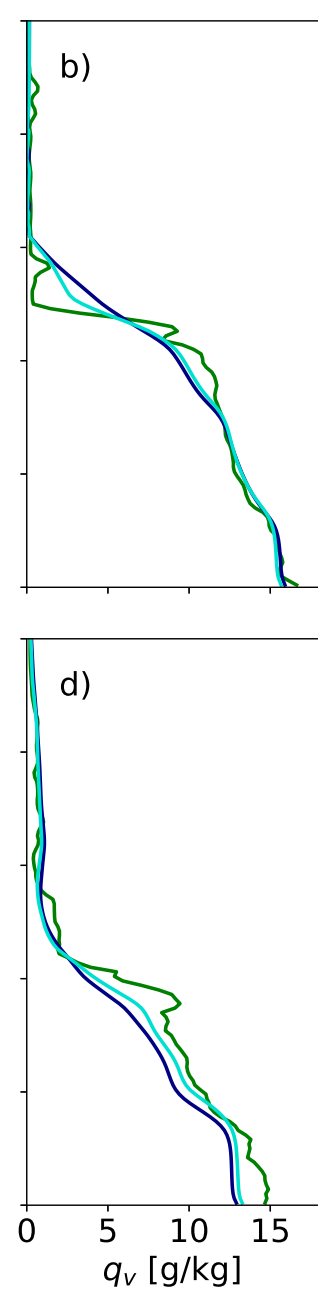

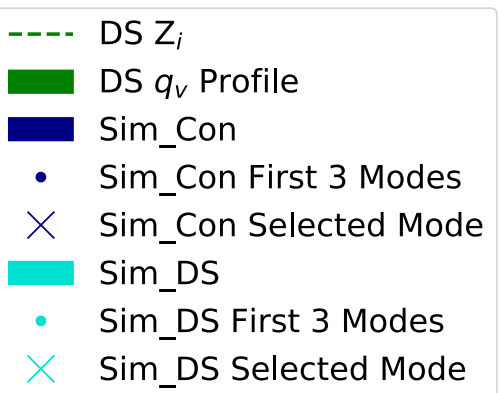

FIG. 12. (a) DS01 and (c) DS03 PDFs of the altitudes at which the maximum $\Delta q_{v}$ occurs for each column in the LES domain for the Sim_Con (navy) and Sim_DS (light blue). The inversion altitude in the corresponding DS profiles is given by the green dashed horizontal line. The dots represent the three largest local maximum number of occurrences, while the crosses represent the LES mode selected by the probabilistic method. (c),(d) Sim_Con (navy) and Sim_DS (light blue) area-averaged profile for $q_{v}$ for the locations and times of DS01 and DS03, respectively. The corresponding $q_{v}$ profiles for the DS are shown in green in (b) and (d).

the LES values is quite significant. In addition, Fig. 14a shows that the inclusion of the DS profile in the forcings appears to only improve the inversion strength in $\Theta_{v}$ in approximately $36 \%$ of the cases. Figure $14 \mathrm{~b}$ shows that the performance for the strength in the humidity inversion is worse, being underestimated by both Sim_Con and Sim_DS. The IFS data also underestimate the inversion strength in $\Theta_{v}$ in $90 \%$ of the cases.

The considerable difference in performance for the strength of the inversion for temperature and humidity is remarkable. We speculate that various reasons for this could exist, including (i) extremely low humidity observed above the trade inversion or (ii) the significantly underestimated inversion strength also in the IFS, seen in Figs. 9b and 14b. Both effects could conspire to make advective forcings in the vicinity of the inversion, as derived from the IFS, less reliable. It could be the case that these forcings play a big role in establishing the strong inversion that is observed. When inversion strength is underestimated in the GCM, sharp differential advection across the inversion is likely not captured. Investigating this possibility and addressing its impact are a future research topic.

\section{f. Sensitivity studies}

An additional 14 simulations were run to determine the sensitivity of the results in relation to different parameters. These parameters include (i) the tightness of the nudging as expressed by $\tau$, (ii) the length of $T$ around $t_{\mathrm{DS}}$, and (iii) the vertical discretization in the LES $(\Delta z)$. Each of these simulations were run for the DS01 location. In the analysis only the lowest $3 \mathrm{~km}$ were 

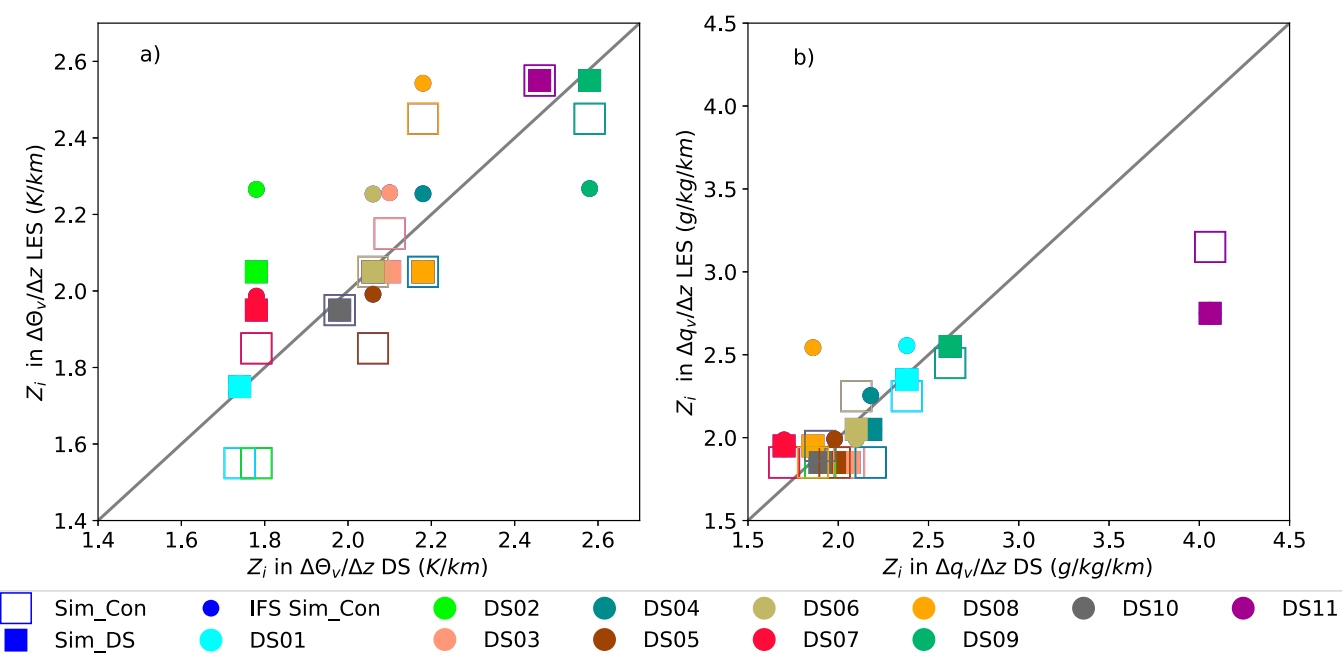

FIG. 13. Comparison between the inversion heights in the DS profile and the height of the selected mode from the inversion-height PDFs for all simulations for (a) $\Theta_{v}$ and (b) $q_{v}$. The unfilled squares represent the modes in the Sim_Con while the filled squares represent the Sim_DS. The inversion heights for the IFS dataset are also included for Sim_Con (IFS Sim_Con) as a reference to where the LES is nudged.

considered, where the DS has the largest impact. The area $A_{q_{v}}$, as defined in Eq. (4), is thus calculated over the lowest $3 \mathrm{~km}$ at $t_{\mathrm{DS}}$. To calculate the area between profiles the DS profile was linearly interpolated onto the respective LES vertical grid.

\section{1) Nudging TIME SCALE}

Ten simulations, divided between Sim_Con and Sim_DS, were carried out to study the impact of increasing and decreasing $\tau$ in the LES. The different $\tau$ values used in this sensitivity study are summarized in Table 2 .
For the purpose of analyzing the impact of varying the value of $\tau$, Sim_Con and Sim_DS for DS01 are used. Figure 15a shows $A_{q_{v}}$ for each value of $\tau$ at $t_{\mathrm{Ds}}$. For each value of $\tau$ the value of $A_{q_{v}}$ for Sim_DS is consistently smaller than that of Sim_Con. Note the slightly larger value for $A_{q_{v}}$, for Sim_Con, when $\tau=1 \mathrm{~h}$ compared to when $\tau>1 \mathrm{~h}$. This deviation is potentially due to the $\tau$ being set to a tight nudging value. The value of $A_{q_{v}}$ does not vary significantly for values of $\tau$ between 2 and $6 \mathrm{~h}$. In the Sim_DS studies, the value of $A_{q_{v}}$ increases with increasing $\tau$. This indicates that the nudging effect
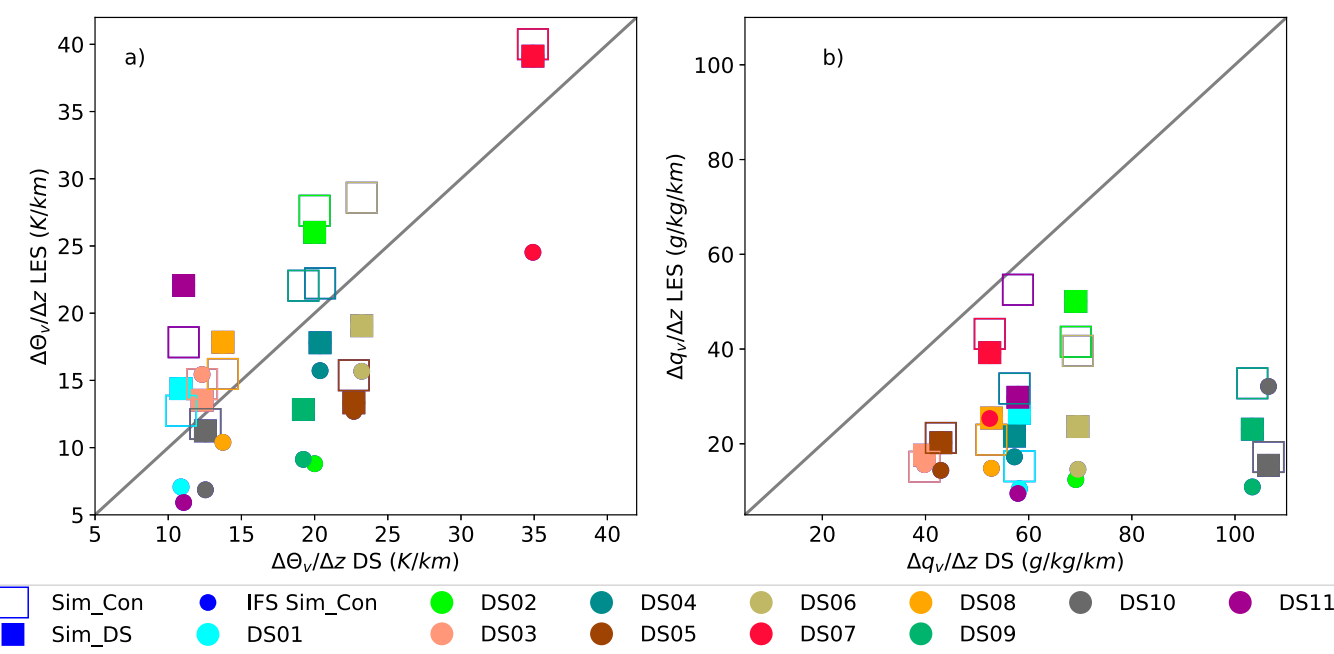

FIG. 14. Comparison of inversion strengths in (a) $\Theta_{v}$ and (b) $q_{v}$ as calculated using Eq. (7) for all DSs (large circles), Sim_Con (unfilled squares), and Sim_DS (filled squares), where individual colors relate both simulations to their corresponding DSs. The inversion strength for the IFS dataset (small circle) are also included for Sim_Con (IFS Sim_Con) as a reference to where the LES is nudged. 

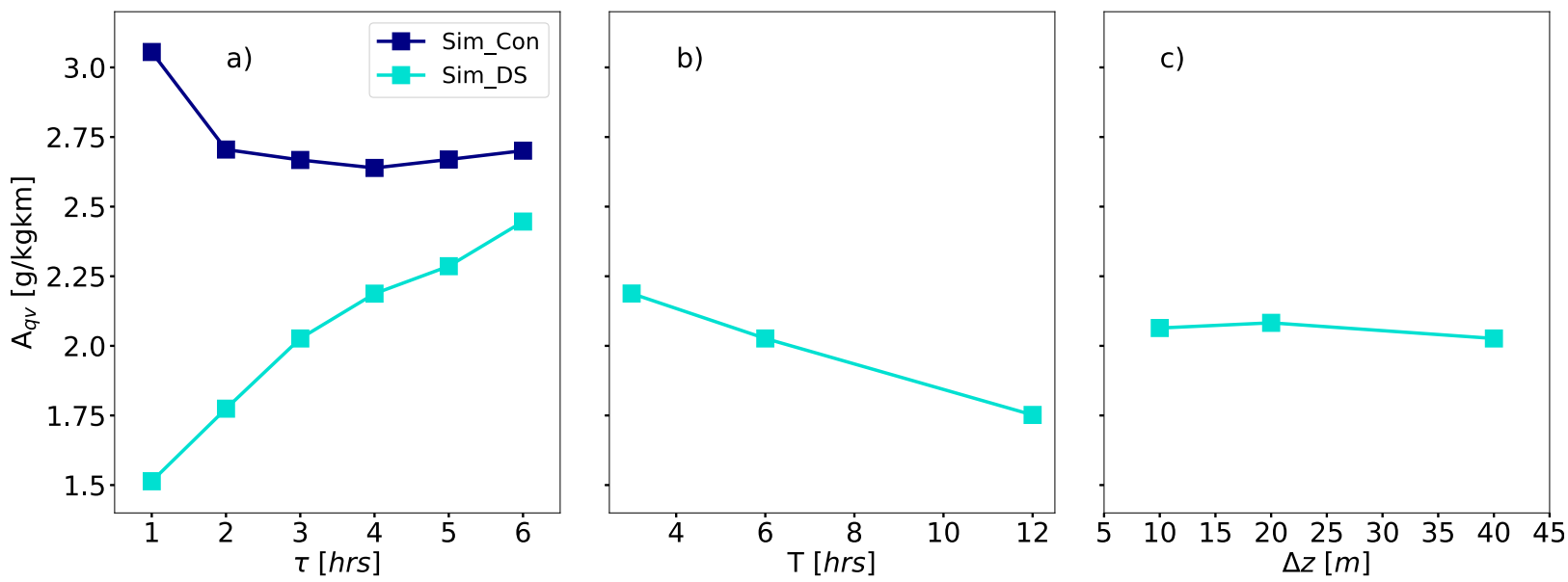

FIG. 15. The area calculated between the observed $q_{v}$ profile and the simulated $q_{v}$ profiles, at the DS launch time, for the sensitivity simulations for (a) the tightness of the nudging as expressed by the nudging time scale $\tau$, (b) the length of the nudging time window $T$, and (c) the vertical resolution $\Delta z$. The Sim_Con (navy) was only run for the sensitivity simulations on the nudging tightness.

decreases with increasing $\tau$. Therefore, it can be determined that the simulation where $\tau$ is equal to $1 \mathrm{~h}$, where the nudging strength is strongest, captures the DS profile with the highest accuracy. However, one issue with using $\tau=1 \mathrm{~h}$ is that the turbulence unable to act freely in the simulation.

\section{2) Nudging TIME WINDOW}

Two simulations were carried out to study the impact of the duration of the time window during which the DS profile affects the IFS dataset. Similar to the experiments conducted for $\tau$, these simulations are summarized in Table 2. Using the method described for the Sim_DS, simulations where $T=3 \mathrm{~h}$ and $T=12 \mathrm{~h}$ were generated. Figure $15 \mathrm{~b}$ shows $A_{q_{v}}$ at $t_{\mathrm{DS}}$ for each value of $T$. The value of $A_{q_{v}}$ is lowest for $T=12 \mathrm{~h}$, and largest for $T=3 \mathrm{~h}$. Therefore, the larger time window makes the nudging more effective allowing the simulation to be more representative of the BL observed.

\section{3) VerticAl RESOLUTION}

The final sensitivity study conducted was on the vertical resolution. As mentioned in the previous sensitivity experiments, Table 2 summarizes the two simulations carried out for this sensitivity study. This study was carried out to investigate whether having a higher vertical resolution would improve the representation of the BL in the atmospheric profiles. Two simulations were carried out in the vertical resolution test, one where $\Delta z=20 \mathrm{~m}$ and a second where $\Delta z=10 \mathrm{~m}$. All LES input files were interpolated onto a higher vertical grid resolution. Figure $15 \mathrm{c}$ shows $A_{q_{v}}$ for each of the studied resolutions. Over the three simulations $A_{q_{v}}$ varies by approximately $0.05 \mathrm{~g} \mathrm{~kg}^{-1} \mathrm{~km}^{-1}$. Therefore, it is determined that there is very little impact in increasing the vertical resolution, for this case.

\section{Discussion}

Throughout this study the LES has shown good skill in reproducing the mean thermodynamic structure, wind speeds, IWV, and inversion heights. A significant impact due to nudging the LES toward DS profiles is seen on the simulated trade wind cumulus fields. Finally, a probabilistic method for comparing observed and simulated inversion properties has been shown to be successful.

There are, however, some shortcomings. For example, the representation of the inversion strength, particularly in humidity. As speculated earlier this could be due to the extremely low values in humidity directly above the inversion, or potentially due to the representation of the inversion in the IFS dataset. Investigating this shortcoming is definitely an important future research topic. A second possible shortcoming is that the use of a limited domain size can artificially boost variability in the mean.

\section{Conclusions}

In this study LES realizations were generated of cumulus cloud fields observed by HALO during NARVAL. The main novelties compared to previous studies of this kind (e.g., Bretherton et al. 1999; Siebesma et al. 2003; vanZanten et al. 2011) include (i) the use of time-varying large-scale forcings and (ii) the incorporation of dropsonde profiles using a simple assimilation method. 
The main results of this study are briefly summarized as follows:

- The simulations show a strong temporal evolution of the boundary layer state, commensurate with previous observational studies at the BCO site.

- Comparing Sim_Con and Sim_DS with the independent observational data from HAMP reveals that domain-averaged LES IWV is representative of the observed median IWV.

- The representation of the atmospheric profiles throughout the boundary layer is improved in Sim_DS compared to Sim_Con. This is due to nudging the LES toward the observed (dropsonde) profiles in the large-scale forcings as opposed to nudging toward a GCM-derived state.

- The probabilistic method for evaluating inversion properties shows that the inversion height is reproduced to a satisfactory degree. However, the simulated inversion strength contains significant spread, and is also significantly underestimated in water vapor.

- As expected, sensitivity tests show considerable dependence on the tightness of the nudging, with the more representative boundary layer profiles in the LES with a nudging time scale of $3 \mathrm{~h}$ compared to $6 \mathrm{~h}$, when the simulations were compared to observed profiles. Also concluded from these sensitivity tests was a weak dependence on vertical resolution, with little difference when the resolution is increased by a factor of 2 .

The method detailed in this study can also be applied to several other field campaigns where the profiles of a number of measured variables can be blended into the large-scale forcings.

In a related and ongoing follow-up study, the clouds in these simulations are evaluated against additional observations recorded by instrumentation on board HALO, for example, HAMP. A key question addressed in that study is how well the observed horizontal variability in the convective cloud field is reproduced by the LES, and how this relates to spatial organization.

Acknowledgments. The research carried out for this study was conducted as part of the High-Definition Clouds and Precipitation for advancing Climate Prediction $\left[\mathrm{HD}(\mathrm{CP})^{2}\right]$ project funded by the German Federal Ministry of Education and Research within the program Research for Sustainable Development (FONA; www.fona.de), under Grant 01LK1504C. The authors thank Dr. Mario Mech and Marek Jacob for their assistance regarding information about HALO and the dropsonde data, and Dr. Jan Chylik for his assistance with the DALES model. The authors would also like to thank the three anonymous reviewers for their helpful comments, which have greatly improved the

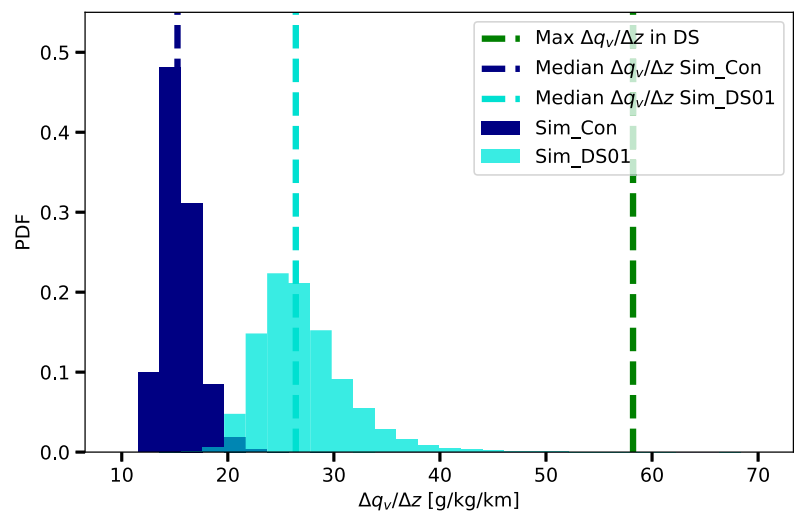

FIG. A1. PDFs of maximum $\Delta q_{v} / \Delta z$ values for the Sim_Con (navy) and Sim_DS01 (light blue), as well as the medians of both simulations represented by the dashed line of the respective colors, and the maximum $\Delta q_{v} / \Delta z$ in DS01 profile represented by the dashed green line.

paper. We would also like to thank the DLR for providing the measurements by HALO during NARVALSouth, which was funded by the DFG SPP project 1294.

\section{APPENDIX}

\section{Determining the Inversion Strength}

As described at the beginning of section $4 \mathrm{e}$, the inversion strength in $q_{v}$ and $\Theta_{v}$ is calculated for every column in the three-dimensional LES field with an inversion height within the selected mode. A PDF of these inversion strengths is produced for each Sim_Con and Sim_DS. The median of these inversion strength PDFs is then compared to the inversion strength in the corresponding DS profile.

Taking DS01 as an example, Figs. A1 and A2 show the PDFs of the inversion strength in $q_{v}$ and $\Theta_{v}$, respectively. The medians of the Sim_Con and Sim_DS01 PDFs, represented by the dashed navy and light blue lines, respectively, are compared with the inversion strength in the DS01 $q_{v}$ and $\Theta_{v}$ profiles, represented by the dashed green line.

From Fig. A1 it is clear that both simulations underestimate the inversion strength compared to the observed inversion strength. While both simulations underestimate the inversion strength, the median of the Sim_DS01 PDF is more representative of the inversion strength in the DS01 $q_{v}$ profile. For the inversion strength in $\Theta_{v}$ comparison Fig. A2 shows that both simulations capture the inversion strength to a relatively high degree. Comparing the median inversion strength in $\Theta_{v}$ for both simulations to the observed inversion strength shows that the Sim_Con has a more representative in this case. This is repeated for the Sim_Con 


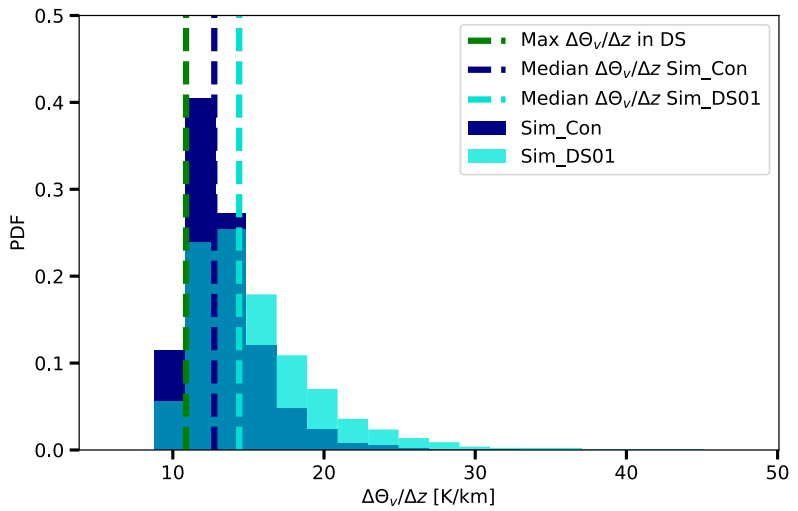

FIG. A2. PDFs of maximum $\Delta \Theta_{v} / \Delta z$ values for the Sim_Con (navy) and Sim_DS01 (light blue), as well as the medians of both simulations represented by the dashed line of the respective colors, and the maximum $\Delta \Theta_{v} / \Delta z$ in DS01 profile represented by the dashed green line.

and Sim_DS at each of the DS locations. The results of the comparisons across all DS locations are seen in section $4 \mathrm{e}$.

\section{REFERENCES}

Ackerman, A. S., and Coauthors, 2009: Large-eddy simulations of a drizzling, stratocumulus-topped marine boundary layer. Mon. Wea. Rev., 137, 1083-1110, https://doi.org/10.1175/ 2008MWR2582.1.

Albrecht, B. A., A. K. Betts, W. H. Schubert, and S. K. Cox, 1979: Model of the thermodynamic structure of the trade-wind boundary layer: Part I. Theoretical formulation and sensitivity tests. J. Atmos. Sci., 36, 73-89, https://doi.org/10.1175/15200469(1979)036<0073:MOTTSO > 2.0.CO;2.

- C. S. Bretherton, D. Johnson, W. H. Scubert, and A. S. Frisch, 1995: The Atlantic Stratocumulus Transition ExperimentASTEX. Bull. Amer. Meteor. Soc., 76, 889-904, https://doi.org/ 10.1175/1520-0477(1995)076<0889:TASTE > 2.0.CO;2.

Bellon, G., and B. Stevens, 2012: Using the sensitivity of largeeddy simulations to evaluate atmospheric boundary layer models. J. Atmos. Sci., 69, 1582-1601, https://doi.org/10.1175/ JAS-D-11-0160.1.

Blossey, P. N., C. S. Bretherton, A. Cheng, S. Endo, T. Heus, A. P. Lock, and J. J. van der Dussen, 2016: CGILS phase 2 LES intercomparison of response of subtropical marine low cloud regimes to $\mathrm{CO}_{2}$ quadrupling and a CMIP3 composite forcing change. J. Adv. Model. Earth Syst., 8, 1714-1726, https:// doi.org/10.1002/2016MS000765.

Bony, S., and J.-L. Dufresne, 2005: Marine boundary layer clouds at the heart of tropical cloud feedback uncertainties in climate models. Geophys. Res. Lett., 32, L20806, https://doi.org/ 10.1029/2005GL023851.

— , and Coauthors, 2015: Clouds, circulation and climate sensitivity. Nat. Geosci., 8, 261-268, https://doi.org/10.1038/ngeo2398.

Bretherton, C. S., S. K. Krueger, M. C. Wyant, P. Bechtold, E. V. Meijgaard, B. Stevens, and J. Teixeira, 1999: A GCSS boundary-layer cloud model intercomparison study of the first ASTEX Lagrangian experiment. Bound.-Layer Meteor., 93, 341-380, https://doi.org/10.1023/A:1002005429969.

Brown, A. R., and Coauthors, 2002: Large-eddy simulation of the diurnal cycle of shallow cumulus convection over land. Quart.
J. Roy. Meteor. Soc., 128, 1075-1093, https://doi.org/10.1256/ 003590002320373210.

Dal Gesso, S., and R. A. J. Neggers, 2018: Can we use singlecolumn models for understanding the boundary layer cloudclimate feedback? J. Adv. Model. Earth Syst., 10, 245-261, https://doi.org/10.1002/2017MS001113.

Deardorff, J. W., 1972: Theoretical expression for the countergradient vertical heat flux. J. Geophys. Res., 77, 5900-5904, https://doi.org/10.1029/JC077i030p05900.

Duynkerke, P. G., and Coauthors, 1999: Intercomparison of threeand one-dimensional model simulations and aircraft observations of stratocumulus. Bound.-Layer Meteor., 92, 453-487, https://doi.org/10.1023/A:1002006919256.

Gentine, P., G. Bellon, and C. C. van Heerwaarden, 2015: A closer look at boundary layer inversion in large-eddy simulations and bulk models: Buoyancy-driven case. J. Atmos. Sci., 72, 728-749, https://doi.org/10.1175/JAS-D-13-0377.1.

Gustafson, W. I., X. Cheng, B. Krishna, T. Toto, A. M. Vogelmann, S. Endo, Z. Li, and H. Xiao, 2017: Description of the LASSO Alpha 1 Release. DOE ARM Climate Research Facility Tech. Rep. DOE/SC-ARM-TR-194, 57 pp., https://doi.org/ 10.2172/1373564.

Heus, T., and Coauthors, 2010: Formulation of the Dutch Atmospheric Large-Eddy Simulation (DALES) and overview of its applications. Geophys. Model Dev., 3, 415-444, https://doi.org/ 10.5194/gmd-3-415-2010.

Hock, T. F., and J. L. Franklin, 1999: The NCAR GPS dropwindsonde. Bull. Amer. Meteor. Soc., 80, 407-420, https:// doi.org/10.1175/1520-0477(1999)080<0407:TNGD> 2.0. $\mathrm{CO} ; 2$.

Hundsdorfer, W., B. Koren, M. vanLoon, and J. Verwer, 1995: A positive finite-difference advection scheme. J. Comput. Phys., 117, 35-46, https://doi.org/10.1006/jcph.1995.1042.

IPCC, 2013: Summary for policymakers. Climate Change 2013: The Physical Science Basis, T. F. Stocker et al., Eds., Cambridge University Press, 1-29.

Jacob, M., F. Ament, M. Gutleben, H. Konow, M. Mech, M. Wirth, and S. Crewell, 2019: Investigating the liquid water path over the tropical Atlantic with synergistic airborne measurements. Atmos. Meas. Tech., 12, 3237-3254, https://doi.org/10.5194/amt-12-3237-2019.

Khairoutdinov, M., and Y. Kogan, 2000: A new cloud physics parameterization in a large-eddy simulation model of marine stratocumulus. Mon. Wea. Rev., 128, 229-243, https://doi.org/ 10.1175/1520-0493(2000)128<0229:ANCPPI > 2.0.CO;2.

Klein, S. A., and D. L. Hartmann, 1993: The seasonal cycle of low stratiform clouds. J. Climate, 6, 1587-1606, https://doi.org/ 10.1175/1520-0442(1993)006<1587:TSCOLS $>2.0$. CO;2.

Klepp, C., F. Ament, S. Bakan, L. Hirsch, and B. Stevens, 2014: NARVAL campaign report. Max Planck Institute of Meteorology Tech. Rep., 218 pp.

Konow, H., and Coauthors, 2018: A unified data set of airborne cloud remote sensing using the HALO Microwave Package (HAMP). Earth Syst. Sci. Data, 11, 921-934, https://doi.org/ 10.5194/essd-11-921-2019.

Lamer, K., P. Kollias, and L. Nuijens, 2015: Observations of the variability of shallow trade wind cumulus cloudiness and mass flux. J. Geophys. Res. Atmos., 120, 6161-6178, https://doi.org/ 10.1002/2014JD022950.

Lilly, D., 1966: The representation of small-scale turbulence in numerical simulation experiments. Proc. IBM Scientific Computing Symp. on Environmental Sciences, Yorktown Heights, NY, IBM, 195-210, https://doi.org/10.5065/d62r3pmm. 
Lock, A. P., 2009: Factors influencing cloud area at the capping inversion for shallow cumulus clouds. Quart. J. Roy. Meteor. Soc., 135, 941-952, https://doi.org/10.1002/qj.424.

Mech, M., E. Orlandi, S. Crewell, F. Ament, L. Hirsch, M. Hagen, G. Peters, and B. Stevens, 2014: HAMP-The microwave package on the High Altitude and Long Range Research Aircraft (HALO). Atmos. Meas. Tech., 7, 4539-4553, https:// doi.org/10.5194/amt-7-4539-2014.

Moeng, C.-H., and Coauthors, 1996: Simulation of a stratocumulustopped planetary boundary layer: Intercomparison among different numerical codes. Bull. Amer. Meteor. Soc., 77, 261-278, https:// doi.org/10.1175/1520-0477(1996)077<0261:SOASTP>2.0.CO;2.

Neggers, R. A. J., 2015: Attributing the behavior of low-level clouds in large-scale models to subgrid-scale parameterizations. J. Adv. Model. Earth Syst., 7, 2029-2043, https://doi.org/ 10.1002/2015MS000503.

_ J. D. Neelin, and B. Stevens, 2007: Impact mechanisms of shallow cumulus convection on tropical climate dynamics. J. Climate, 20, 2623-2642, https://doi.org/10.1175/JCLI4079.1.

— A. P. Siebesma, and T. Heus, 2012: Continuous single-column model evaluation at a permanent meteorological supersite. Bull. Amer. Meteor. Soc., 93, 1389-1400, https://doi.org/ 10.1175/BAMS-D-11-00162.1.

_ , and Coauthors, 2017: Single-column model simulations of subtropical marine boundary-layer cloud transitions under weakening inversions. J. Adv. Model. Earth Syst., 9, 23852412, https://doi.org/10.1002/2017MS001064.

Nuijens, L., and B. Stevens, 2012: The influence of wind speed on shallow marine cumulus convection. J. Atmos. Sci., 69, 168184, https://doi.org/10.1175/JAS-D-11-02.1.

— I. Serikov, L. Hirsch, K. Lonitz, and B. Stevens, 2014: The distribution and variability of low-level cloud in the North Atlantic trades. Quart. J. Roy. Meteor. Soc., 140, 2364-2374, https://doi.org/10.1002/qj.2307.

Rauber, R. M., and Coauthors, 2007: Rain in Shallow Cumulus Over the Ocean: The RICO campaign. Bull. Amer. Meteor. Soc., 88, 1912-1928, https://doi.org/10.1175/BAMS-88-12-1912.

Rieck, M., L. Nuijens, and B. Stevens, 2012: Marine boundary layer cloud feedbacks in a constant relative humidity atmosphere. J. Atmos. Sci., 69, 2538-2550, https://oi.org/10.1175/ JAS-D-11-0203.1.

Schnitt, S., E. Orlandi, M. Mech, A. Ehrlich, and S. Crewell, 2017: Characterization of water vapor and clouds during the NextGeneration Aircraft Remote Sensing for Validation (NARVAL) south studies. IEEE J. Sel. Top. Appl. Earth Obs. Remote Sens., 10, 3114-3124, https://doi.org/10.1109/JSTARS.2017.2687943.

Seifert, A., and K. D. Beheng, 2006: A two-moment cloud microphysics parameterization for mixed-phase clouds. Part 1: Model description. Meteor. Atmos. Phys., 92, 45-66, https://doi.org/ 10.1007/s00703-005-0112-4.
— , and T. Heus, 2013: Large-eddy simulation of organized precipitating trade wind cumulus clouds. Atmos. Chem. Phys., 13, 5631-5645, https://doi.org/10.5194/acp-13-5631-2013.

Siebesma, A. P., and Coauthors, 2003: A large eddy simulation intercomparison study of shallow cumulus convection. J. Atmos. Sci., 60, 1201-1219, https://doi.org/10.1175/15200469(2003)60<1201:ALESIS>2.0.CO;2.

Sommeria, G., 1976: Three-dimensional simulation of turbulent processes in an undisturbed trade wind boundary layer. J. Atmos. Sci., 33, 216-241, https://doi.org/10.1175/15200469(1976)033<0216:TDSOTP $>2.0$. CO;2.

Stevens, B., and Coauthors, 2001: Simulations of trade wind cumuli under a strong inversion. J. Atmos. Sci., 58, 18701891, https://doi.org/10.1175/1520-0469(2001)058<1870: SOTWCU $>2.0 . \mathrm{CO} ; 2$.

_ , and Coauthors, 2016: The Barbados Cloud Observatory: Anchoring investigations of clouds and circulation on the edge of the ITCZ. Bull. Amer. Meteor. Soc., 97, 787-801, https:// doi.org/10.1175/BAMS-D-14-00247.1.

_ C.-H. Moeng, and P. P. Sullivan, 1999: Large-eddy simulations of radiatively driven convection: Sensitivities to the representation of small scales. J. Atmos. Sci., 56, 3963-3984, https://doi.org/10.1175/ 1520-0469(1999)056<3963:LESORD > 2.0.CO;2.

Stull, R. B., 1988: An Introduction to Boundary Layer Meteorology. Kluwer Academic, 666 pp.

Tan, Z., T. Schneider, J. Teixeira, and K. G. Pressel, 2016: Large-eddy simulation of subtropical cloud-topped boundary layers: 1. A forcing framework with closed surface energy balance. $J$. Adv. Model. Earth Syst., 8, 1565-1585, https://doi.org/10.1002/2016MS000655.

Teixeira, J., and Coauthors, 2008: Parameterization of the atmospheric boundary layer: A view from just above the inversion. Bull. Amer. Meteor. Soc., 89, 453-458, https://doi.org/10.1175/ BAMS-89-4-453.

van der Dussen, J. J., and Coauthors, 2013: The GASS/EUCLIPSE model intercomparison of the stratocumulus transition as observed during ASTEX: LES results. J. Adv. Model. Earth Syst., 5, 483-499, https://doi.org/10.1002/jame.20033.

van Laar, T., V. Schemann, and R. A. J. Neggers, 2019: Investigating the diurnal evolution of the cloud size distribution of continental cumulus convection using multi-day LES. J. Atmos. Sci., 76, 729-747, https://doi.org/10.1175/JAS-D-18-0084.1

vanZanten, M. C., and Coauthors, 2011: Controls on precipitation and cloudiness in simulations of trade-wind cumulus as observed during RICO. J. Adv. Model. Earth Syst., 3, M06001, https://doi.org/10.1029/2011MS000056.

Wood, R., and C. S. Bretherton, 2004: Boundary layer depth, entrainment, and decoupling in the cloud-capped subtropical and tropical marine boundary layer. J. Climate, 17, 3576-3588, https://doi.org/10.1175/1520-0442(2004)017<3576: BLDEAD $>2.0 . \mathrm{CO} ; 2$. 